\title{
FULL AND PARTIAL CLOAKING IN ELECTROMAGNETIC SCATTERING
}

\author{
YOUJUN DENG, HONGYU LIU, AND GUNTHER UHLMANN
}

\begin{abstract}
In this paper, we consider two regularized transformation-optics cloaking schemes for electromagnetic (EM) waves. Both schemes are based on the blowup construction with the generating sets being, respectively, a generic curve and a planar subset. We derive sharp asymptotic estimates in assessing the cloaking performances of the two constructions in terms of the regularization parameters and the geometries of the cloaking devices. The first construction yields an approximate full-cloak, whereas the second construction yields an approximate partial-cloak. Moreover, by incorporating properly chosen conducting layers, both cloaking constructions are capable of nearly cloaking arbitrary EM contents. This work complements the existing results in $5-7$ on approximate EM cloaks with the generating set being a singular point, and it also extends 925 on regularized full and partial cloaks for acoustic waves governed by the Helmholtz system to the more challenging EM case governed by the full Maxwell system.
\end{abstract}

Keywords: Maxwell equations, invisibility cloaking, transformation optics, partial and full cloaks, regularization, asymptotic estimates

2010 Mathematics Subject Classification: 35Q60, 31B10, 35R30, 78A40

\section{INTRODUCTION}

1.1. Background. This paper concerns with invisibility cloaking of electromagnetic (EM) waves via the approach of transformation optics. This method which is based on the transformation properties of the optical material parameters and the invariance properties of the equations modeling the wave phenomena, was pioneered in [16] for electrostatics. For Maxwell's system, the same transformation optics approach was developed in [34]. In two dimensions, Leonhardt [24] used the conformal mapping to cloak rays in the geometric optics approximation. The obtained cloaking materials are called metamaterials. The metamaterials proposed in [17,24,34 for the ideal cloaks are singular, and this has aroused great interest in the literature to deal with the singular structures. In [12,30], the authors proposed to consider the finite-energy solutions from singularly weighted Sobolev spaces for the underlying singular PDEs, and both acoustic cloaking and EM cloaking were treated. In [11, 13, 20, 21, 27], the authors proposed to avoid the singular structures by incorporating regularization into the cloaking construction, and instead of ideal 
invisibility cloak, one considers approximate/near invisibility cloak. The latter approach has been further investigated in $3,4,28]$ for the conductivity equation and Helmholtz system, modeling electric impedance tomography (EIT) and acoustic wave scattering, respectively; and in [5-7] for the Maxwell system, modeling the EM wave scattering. For all of the above mentioned work on regularized approximate cloaks, the generating set is a singular point, and one always achieves the full-cloak; that is, the invisibility is attainable for detecting waves coming from every possible incident/impinging direction, and observations made at every possible angle. In a recent article [25], the authors proposed to study regularized partial/customized cloaks for acoustic waves; that is, the invisibility is only attainable for limited/customized apertures of incidence and observation. The key idea is to properly choose the generating set for the blowup construction of the cloaking device. In [25], the authors only proved qualitative convergence for the proposed partial/customized cloaking construction, and the corresponding result was further quantified in [9] by the authors of the current article.

In this paper, we shall extend 9,25 on the regularized partial/customized cloaks for acoustic wave scattering to the case of electromagnetic wave scattering. We present two near-cloaking schemes with the generating sets being a generic curve or a planar subset, respectively. It is shown that the first scheme yields an approximate full-cloak, whose invisibility effect is attainable for the whole aperture of incidence and observation angles. The result obtained complements [5 7] on approximate full cloaks. However, the generating set for the blowup construction considered in [5-7] is a singular point, whereas in this study, the generating set is a generic curve. The cloaking material in our full-cloaking scheme is less "singular" than those in [5 7], but at the cost of losing some degree of accuracy on the invisibility approximation. The second scheme would yield an approximate partial-cloak with limited apertures of incidence and observation. We derive sharp asymptotic estimates in assessing the cloaking performances in terms of the regularization parameters and the geometries of the generating sets. The estimates are independent of the EM contents being cloaked, which means that the proposed cloaking schemes are capable of nearly cloaking arbitrary EM objects. Compared to [5 7] on approximate full-cloaks, we need to deal with anisotropic geometries, whereas compared to [9, 25] on approximate partial-cloaks for acoustic scattering governed by the Helmholtz system, we need to tackle the more challenging Maxwell system. Finally, we refer the readers to $8,14,15,29,37$ for surveys on the theoretical and experimental progress on transformation-optics cloaking in the literature.

1.2. Mathematical formulation. Consider a homogeneous space with the (normalized) EM medium parameters described by the electric permittivity $\varepsilon_{0}=\mathbf{I}_{3 \times 3}$ and magnetic permeability $\mu_{0}=\mathbf{I}_{3 \times 3}$. Here and also in what follows, $\mathbf{I}_{3 \times 3}$ denotes the identity matrix in $\mathbb{R}^{3 \times 3}$. For notational convenience, we also let $\sigma_{0}:=0 \cdot \mathbf{I}_{3 \times 3}$ denote the conductivity tensor of the homogeneous background space. We shall 
consider the invisibility cloaking in the homogeneous space described above. Following the spirt in [5] 7 , the proposed cloaking device is compactly supported in a bounded domain $\Omega$, and takes a three-layered structure. Let $\Omega_{a} \Subset \Omega_{c} \Subset \Omega$ be bounded domains such that $\Omega_{a}, \Omega_{c} \backslash \bar{\Omega}_{a}$ and $\Omega \backslash \bar{\Omega}_{c}$ are connected, and they represent, respectively, the cloaked region, conducting layer and cloaking layer of the proposed cloaking device. Let $\Gamma_{0}$ be a bounded open set in $\mathbb{R}^{3}$, and it shall be referred to as a generating set in the following. For $\delta \in \mathbb{R}_{+}$, we let $D_{\delta}$ denote an open neighborhood of $\Gamma_{0}$ such that $D_{\delta} \rightarrow \Gamma_{0}$ (in the sense of Hausdorff distance) as $\delta \rightarrow+0$. $D_{\delta}$ will be referred to as the virtual domain, and shall be specified below. Throughout, we assume that there exists a bi-Lipschitz and orientation-preserving mapping $F_{\delta}: \mathbb{R}^{3} \rightarrow \mathbb{R}^{3}$ such that

$F_{\delta}\left(D_{\delta / 2}\right)=\Omega_{a}, F_{\delta}\left(D_{\delta} \backslash \bar{D}_{\delta / 2}\right)=\Omega_{c} \backslash \bar{\Omega}_{a}, F_{\delta}\left(\Omega \backslash \bar{D}_{\delta}\right)=\Omega \backslash \bar{\Omega}_{c}$ and $\left.F_{\delta}\right|_{\mathbb{R}^{3} \backslash \Omega}=$ Identity

Next, we describe the EM medium parameter distributions $\left\{\mathbb{R}^{3} ; \varepsilon, \mu, \sigma\right\}$ in the physical space containing the cloaking device, and $\left\{\mathbb{R}^{3} ; \varepsilon_{\delta}, \mu_{\delta}, \sigma_{\delta}\right\}$ in the virtual space containing the virtual domain. The EM medium parameters are all assumed to be symmetric-positive-definite-matrix valued functions, and they characterize, respectively, the electric permittivity, magnetic permeability and electric conductivity. In what follows, $\left\{\mathbb{R}^{3} ; \varepsilon, \mu, \sigma\right\}$ and $\left\{\mathbb{R}^{3} ; \varepsilon_{\delta}, \mu_{\delta}, \sigma_{\delta}\right\}$ shall be referred to as, respectively, the physical and virtual scattering configurations. Let

$$
\left\{\mathbb{R}^{3} ; \varepsilon, \mu, \sigma\right\}=\left\{\begin{array}{lll}
\varepsilon_{0}, \mu_{0}, \sigma_{0} & \text { in } \mathbb{R}^{3} \backslash \bar{\Omega}, \\
\varepsilon_{c}^{*}, \mu_{c}^{*}, \sigma_{c}^{*} \text { in } \Omega \backslash \bar{\Omega}_{c}, \\
\varepsilon_{l}^{*}, \mu_{l}^{*}, \sigma_{l}^{*} \text { in } \Omega_{c} \backslash \bar{\Omega}_{a}, \\
\varepsilon_{a}^{*}, \mu_{a}^{*}, \sigma_{a}^{*} \text { in } \Omega_{a},
\end{array}\right.
$$

and

$$
\left\{\mathbb{R}^{3} ; \varepsilon_{\delta}, \mu_{\delta}, \sigma_{\delta}\right\}=\left\{\begin{array}{lll}
\varepsilon_{0}, \mu_{0}, \sigma_{0} & \text { in } & \mathbb{R}^{3} \backslash \bar{D}_{\delta}, \\
\varepsilon_{l}, \mu_{l}, \sigma_{l} & \text { in } & D_{\delta} \backslash \bar{D}_{\delta / 2}, \\
\varepsilon_{a}, \mu_{a}, \sigma_{a} & \text { in } & D_{\delta / 2} .
\end{array}\right.
$$

The virtual and physical scattering configurations are connected by the so-called push-forward via the (blowup) transformation $F_{\delta}$ in (1.1). To that end, we next introduce the push-forward of EM mediums. Let $m$ and $m_{\delta}$, respectively, denote the physical and virtual parameter tensors, where $m=\varepsilon, \mu$ or $\sigma$. Define the push-forward $\left(F_{\delta}\right)_{*} m_{\delta}$ as

$$
m=\left(F_{\delta}\right)_{*} m_{\delta}:=\left(\frac{1}{\operatorname{det}\left(D F_{\delta}\right)}\left(D F_{\delta}\right) \cdot m_{\delta} \cdot\left(D F_{\delta}\right)^{T}\right) \circ F_{\delta}^{-1},
$$

where $D F_{\delta}$ denotes the Jacobian matrix of the transformation $F_{\delta}$. Throughout the rest of our study, we assume that

$$
\left\{\mathbb{R}^{3} ; \varepsilon, \mu, \sigma\right\}=\left(F_{\delta}\right)_{*}\left\{\mathbb{R}^{3} ; \varepsilon_{\delta}, \mu_{\delta}, \sigma_{\delta}\right\}:=\left\{\mathbb{R}^{3} ;\left(F_{\delta}\right)_{*} \varepsilon_{\delta},\left(F_{\delta}\right)_{*} \mu_{\delta},\left(F_{\delta}\right)_{*} \sigma_{\delta}\right\} .
$$


Next, we consider the time-harmonic EM wave scattering in the physical space. Let

$$
\mathbf{E}^{i}(\mathbf{x}):=\mathbf{p} e^{i \omega \mathbf{x} \cdot \mathbf{d}}, \quad \mathbf{H}^{i}:=\frac{1}{\omega}\left(\nabla \times \mathbf{E}^{i}\right)(\mathbf{x}), \quad \mathbf{x} \in \mathbb{R}^{3},
$$

where $\mathbf{p} \in \mathbb{R}^{3} \backslash\{0\}, \mathbf{d} \in \mathbb{S}^{2}$ and $\omega \in \mathbb{R}_{+} \cdot\left(\mathbf{E}^{i}, \mathbf{H}^{i}\right)$ in 1.6$)$ is called a pair of EM plane waves with $\mathbf{E}^{i}$ the electric field and $\mathbf{H}^{i}$ the magnetic field. $\mathbf{p}$ is the polarization tensor, $\mathbf{d}$ is the incident direction and $\omega$ is the wavenumber of the plane waves $\mathbf{E}^{i}$ and $\mathbf{H}^{i}$. There always holds that

$$
\mathbf{p} \perp \mathbf{d}, \quad \text { namely } \mathbf{p} \cdot \mathbf{d}=0 .
$$

$\mathbf{E}^{i}$ and $\mathbf{H}^{i}$ are entire solutions to the following Maxwell equations

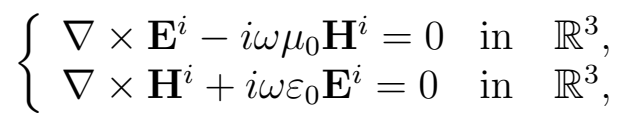

The EM scattering in the physical space $\left\{\mathbb{R}^{3} ; \varepsilon, \mu, \sigma\right\}$ due to the incident plane waves $\left(\mathbf{E}^{i}, \mathbf{H}^{i}\right)$ is described by the following Maxwell system

$$
\begin{cases}\nabla \times \mathbf{E}-i \omega \mu \mathbf{H}=0 & \text { in } \mathbb{R}^{3} \\ \nabla \times \mathbf{H}+i \omega\left(\varepsilon+i \frac{\sigma}{\omega}\right) \mathbf{E}=0 & \text { in } \mathbb{R}^{3}\end{cases}
$$

subject to the Silver-Müller radiation condition:

$$
\lim _{\|\mathbf{x}\| \rightarrow \infty}\|\mathbf{x}\|\left(\left(\mathbf{H}-\mathbf{H}^{i}\right) \times \hat{\mathbf{x}}-\left(\mathbf{E}-\mathbf{E}^{i}\right)\right)=0,
$$

where $\hat{\mathbf{x}}=\mathbf{x} /\|\mathbf{x}\|$ for $\mathbf{x} \in \mathbb{R}^{3} \backslash\{0\}$. We seek solutions $\mathbf{E}, \mathbf{H} \in H_{\text {loc }}\left(\operatorname{curl} ; \mathbb{R}^{3}\right)$ to (1.8); see 19, 22, 23, 31 for the well-posedness of the scattering system (1.8). Here and also in what follows, we shall often use the spaces

$$
H_{\text {loc }}(\operatorname{curl} ; X)=\left\{\left.U\right|_{B} \in H(\operatorname{curl} ; B) ; B \text { is any bounded subdomain of } X\right\}
$$

and

$$
H(\operatorname{curl} ; B)=\left\{U \in\left(L^{2}(B)\right)^{3} ; \nabla \times U \in\left(L^{2}(B)\right)^{3}\right\} .
$$

It is known that the solution $\mathbf{E}$ to $(1.8)$ admits the following asymptotic expansion as $\|\mathbf{x}\| \rightarrow \infty$ (see, e.g., $10 \|$ )

$$
\mathbf{E}(\mathbf{x})-\mathbf{E}^{i}(\mathbf{x})=\frac{e^{i \omega\|\mathbf{x}\|}}{\|\mathbf{x}\|} \mathbf{A}_{\infty}\left(\frac{\mathbf{x}}{\|\mathbf{x}\|} ; \mathbf{E}^{i}\right)+\mathcal{O}\left(\frac{1}{\|\mathbf{x}\|^{2}}\right)
$$

where

$$
\mathbf{A}_{\infty}(\hat{\mathbf{x}} ; \mathbf{p}, \mathbf{d}):=\mathbf{A}_{\infty}\left(\frac{\mathbf{x}}{\|\mathbf{x}\|} ; \mathbf{E}^{i}\right)
$$

is known as the scattering amplitude and $\hat{\mathbf{x}}$ denotes the direction of observation. It is readily verified that

$$
\mathbf{A}_{\infty}(\hat{\mathbf{x}} ; \mathbf{p}, \mathbf{d})=\|\mathbf{p}\| \mathbf{A}_{\infty}\left(\hat{\mathbf{x}} ; \frac{\mathbf{p}}{\|\mathbf{p}\|}, \mathbf{d}\right) .
$$

Therefore, without loss of generality and throughout the rest of our study, we shall assume that $\|\mathbf{p}\|=1$, namely, $\mathbf{p} \in \mathbb{S}^{2}$. 
Definition 1.1. Let $\Sigma_{p} \subset \mathbb{S}^{2}, \Sigma_{d} \subset \mathbb{S}^{2}$ and $\Sigma_{\hat{x}} \subset \mathbb{S}^{2} .\{\Omega ; \varepsilon, \mu, \sigma\}$ is said to be a near/approximate-cloak if

$$
\left\|\mathbf{A}_{\infty}(\hat{\mathbf{x}} ; \mathbf{p}, \mathbf{d})\right\| \ll 1 \text { for } \hat{\mathbf{x}} \in \Sigma_{\hat{x}}, \mathbf{p} \in \Sigma_{p}, \mathbf{d} \in \Sigma_{d} .
$$

If $\Sigma_{p}=\Sigma_{d}=\Sigma_{\hat{x}}=\mathbb{S}^{2}$, then it is called an approximate full-cloak, otherwise it is called an approximate partial-cloak.

According to Definition 1.1, the cloaking layer $\left\{\Omega \backslash \bar{\Omega}_{c} ; \varepsilon_{c}^{*}, \mu_{c}^{*}, \sigma_{c}^{*}\right\}$ together with the conducting layer $\left\{\Omega_{c} \backslash \bar{\Omega}_{a} ; \varepsilon_{l}^{*}, \mu_{l}^{*}, \sigma_{l}^{*}\right\}$ makes the target EM object $\left\{\Omega_{a} ; \varepsilon_{a}^{*}, \mu_{a}^{*}, \sigma_{a}^{*}\right\}$ nearly invisible to detecting waves (1.6) with $\mathbf{d} \in \Sigma_{d}$ and $\mathbf{p} \in \Sigma_{p}$, and observation in the aperture $\Sigma_{\hat{x}}$. $\Sigma_{d}$ and $\Sigma_{\hat{x}}$ shall be referred to as, respectively, the apertures of incidence and observation of the partial-cloaking device. For practical considerations, throughout the current study, we assume that the cloaking device is not object-dependent; that is, the cloaked content $\left\{\Omega_{a} ; \varepsilon_{a}^{*}, \mu_{a}^{*}, \sigma_{a}^{*}\right\}$ is arbitrary but regular, namely, $\varepsilon_{a}^{*}, \mu_{a}^{*}, \sigma_{a}^{*}$ are all arbitrary symmetric positive definite matrices. In Definition 1.1. (1.13) is rather qualitative, and in the subsequent study, we shall quantify the near-cloaking effect and derive sharp estimate in assessing the cloaking performance. To that end, the following theorem plays a critical role (cf. [6, 7]).

Theorem 1.1. Let $(\mathbf{E}, \mathbf{H}) \in H_{l o c}\left(\mathrm{curl} ; \mathbb{R}^{3}\right)^{2}$ be the (unique) pair of solutions to (1.8). Define the pull-back fields by

$$
\mathbf{E}_{\delta}=\left(F_{\delta}\right)^{*} \mathbf{E}:=\left(D F_{\delta}\right)^{T} \mathbf{E} \circ F_{\delta}, \quad \mathbf{H}_{\delta}=\left(F_{\delta}\right)^{*} \mathbf{H}:=\left(D F_{\delta}\right)^{T} \mathbf{H} \circ F_{\delta} .
$$

Then the pull-back fields $\left(\mathbf{E}_{\delta}, \mathbf{H}_{\delta}\right) \in H_{\text {loc }}\left(\operatorname{curl} ; \mathbb{R}^{3}\right)^{2}$ satisfy the following Maxwell equations

$$
\left\{\begin{array}{lll}
\nabla \times \mathbf{E}_{\delta}-i \omega \mu_{0} \mathbf{H}_{\delta}=0 & \text { in } & \mathbb{R}^{3} \backslash \bar{D}_{\delta} \\
\nabla \times \mathbf{H}_{\delta}+i \omega \varepsilon_{0} \mathbf{E}_{\delta}=0 & \text { in } \quad \mathbb{R}^{3} \backslash \bar{D}_{\delta} \\
\nabla \times \mathbf{E}_{\delta}-i \omega \mu_{\delta} \mathbf{H}_{\delta}=0 & \text { in } & D_{\delta} \\
\nabla \times \mathbf{H}_{\delta}+i \omega\left(\varepsilon_{\delta}+i \frac{\sigma_{\delta}}{\omega}\right) \mathbf{E}_{\delta}=0 & \text { in } & D_{\delta}
\end{array}\right.
$$

subject to the Silver-Müller radiation condition:

$$
\lim _{\|\mathbf{x}\| \rightarrow \infty}\|\mathbf{x}\|\left(\left(\mathbf{H}_{\delta}-\mathbf{H}^{i}\right) \times \hat{\mathbf{x}}-\left(\mathbf{E}_{\delta}-\mathbf{E}^{i}\right)\right)=0,
$$

Particularly, since $F_{\delta}=$ Identity in $\mathbb{R}^{3} \backslash \Omega$, one has that

$$
\mathbf{A}_{\infty}\left(\hat{\mathbf{x}} ; \mathbf{E}^{i}\right)=\mathbf{A}_{\infty}^{\delta}\left(\hat{\mathbf{x}} ; \mathbf{E}^{i}\right), \quad \hat{\mathbf{x}} \in \mathbb{S}^{2},
$$

where $\mathbf{A}_{\infty}^{\delta}\left(\hat{\mathbf{x}} ; \mathbf{E}^{i}\right)$ denotes the scattering amplitude corresponding to the Maxwell system (1.14).

Hence, by Theorem 1.1, in order to assess the cloaking performance of the cloaking device $\{\Omega ; \varepsilon, \mu, \sigma\}$ in $(1.2)$ associated with the physical scattering system 
(1.8)-(1.9), it suffices for us to investigate the virtual scattering system (1.14)(1.15). Here, it is noted for emphasis that by (1.5), one has

$$
\left\{D_{\delta / 2} ; \varepsilon_{a}, \mu_{a}, \sigma_{a}\right\}=\left(F_{\delta}^{-1}\right)_{*}\left\{\Omega_{a} ; \varepsilon_{a}^{*}, \mu_{a}^{*}, \sigma_{a}^{*}\right\} .
$$

Since $\left\{\Omega_{a} ; \varepsilon_{a}^{*}, \mu_{a}^{*}, \sigma_{a}^{*}\right\}$ is arbitrary and regular and $F_{\delta}$ is bi-Lipschitz and orientationpreserving, it is clear that $\left\{D_{\delta / 2} ; \varepsilon_{a}, \mu_{a}, \sigma_{a}\right\}$ is also arbitrary and regular.

In summarizing our discussion so far, in order to construct a near-cloaking device, one needs to firstly select a suitable generating set $\Gamma_{0}$ and the corresponding virtual domain $D_{\delta}$; and secondly the blowup transformation $F_{\delta}$ in 1.1); and thirdly the virtual conducting layer $\left\{D_{\delta} \backslash \bar{D}_{\delta / 2} ; \varepsilon_{l}, \mu_{l}, \sigma_{l}\right\}$; and finally assess the cloaking performance by studying the virtual scattering system (1.14)-1.15). In this article, we shall be mainly concerned with extending the full- and partial-cloaking schemes proposed in 9,25 for acoustic waves to the more challenging case with electromagnetic waves. There the generating sets are either a generic curve or a planar subset, and the blowup transformations are constructed via a concatenating technique. Hence, in the current study, we shall mainly focus on properly designing the suitable conducting layers and then assessing the cloaking performances by studying the corresponding virtual scattering system (1.14)-(1.15).

The rest of the paper is organized as follows. In Section 2, we collect some preliminary knowledge on boundary layer potentials, which shall be used throughout our study. Sections 3 and 4 are, respectively, devoted to the study of the full- and partial-cloaking schemes.

\section{BOUNDARY LAYER POTENTIALS}

Our study shall heavily rely on the vectorial boundary integral operators for Maxwell's equations. In this section, we review some of the important properties of the vectorial boundary integral operators for the later use.

2.1. Definitions. Let $D$ be a bounded domain in $\mathbb{R}^{3}$ with a $C^{3}$-smooth boundary $\partial D$ and a connected complement $\mathbb{R}^{3} \backslash \bar{D}$. Let $\nabla_{\partial D}$. denote the surface divergence on $\partial D$ and $\mathrm{H}^{s}(\partial D)$ be the usual Sobolev space of order $s \in \mathbb{R}$ on $\partial D$. Let $\nu$ be the exterior unit normal vector to $\partial D$ and denote by $\mathrm{TH}^{s}(\partial D):=\left\{\mathbf{a} \in \mathrm{H}^{s}(\partial D)^{3} ; \nu \cdot \mathbf{a}=\right.$ $0\}$, the space of vectors tangential to $\partial D$ which is a subset of $\mathrm{H}^{s}(\partial D)^{3}$. We also introduce the function space

$$
\mathrm{TH}_{\text {div }}^{s}(\partial D):=\left\{\mathbf{a} \in \mathrm{TH}^{s}(\partial D) ; \nabla_{\partial D} \cdot \mathbf{a} \in \mathrm{H}^{s}(\partial D)\right\},
$$

endowed with the norm

$$
\|\mathbf{a}\|_{\mathrm{TH}_{\text {div }}^{s}(\partial D)}=\|\mathbf{a}\|_{\mathrm{TH}^{s}(\partial D)}+\left\|\nabla_{\partial D} \cdot \mathbf{a}\right\|_{\mathrm{H}^{s}(\partial D)}
$$


Next, we recall that, for $\omega \in \mathbb{R}_{+} \cup\{0\}$, the fundamental outgoing solution $G_{\omega}$ to the $\operatorname{PDO}\left(\Delta+\omega^{2}\right)$ in $\mathbb{R}^{3}$ is given by

$$
G_{\omega}(\mathbf{x})=-\frac{e^{i \omega\|\mathbf{x}\|}}{4 \pi\|\mathbf{x}\|}
$$

In what follows, if $\omega=0$ we simply write $G_{\omega}$ as $G$.

For a density function $\mathbf{a} \in \mathrm{TH}_{\text {div }}^{s}(\partial D)$, we define the vectorial single layer potential associated with the fundamental solution $G_{\omega}$ introduced in (2.1) by

$$
\mathcal{A}_{D}^{\omega}[\mathbf{a}](\mathbf{x}):=\int_{\partial D} G_{\omega}(\mathbf{x}-\mathbf{y}) \mathbf{a}(\mathbf{y}) d \sigma_{y}, \quad \mathbf{x} \in \mathbb{R}^{3} .
$$

For a scalar density $\varphi \in \mathrm{H}^{s}(\partial D)$, the single layer potential is defined similarly by

$$
\mathcal{S}_{D}^{\omega}[\varphi](\mathbf{x}):=\int_{\partial D} G_{\omega}(\mathbf{x}-\mathbf{y}) \varphi(\mathbf{y}) d \sigma_{y}, \quad \mathbf{x} \in \mathbb{R}^{3} .
$$

The following boundary operator shall also be needed

$$
\begin{aligned}
\mathcal{M}_{D}^{\omega}: \mathrm{L}_{T}^{2}(\partial D) & \longrightarrow \mathrm{L}_{T}^{2}(\partial D) \\
\mathbf{a} & \longrightarrow \mathcal{M}_{D}^{\omega}[\mathbf{a}](\mathbf{x})=\text { p.v. } \quad \nu_{\mathbf{x}} \times \nabla \times \int_{\partial D} G_{\omega}(\mathbf{x}, \mathbf{y}) \mathbf{a}(\mathbf{y}) d \sigma_{y},
\end{aligned}
$$

where $\mathrm{L}_{T}^{2}(\partial D):=\mathrm{TH}^{0}(\partial D)$, and p.v. signifies the Cauchy principle value. In what follows, we denote by $\mathcal{A}_{D}, \mathcal{S}_{D}$ and $\mathcal{M}_{D}$ the operators $\mathcal{A}_{D}^{0}, \mathcal{S}_{D}^{0}$ and $\mathcal{M}_{D}^{0}$, respectively.

2.2. Boundary integral identities. Here and throughout the rest of the paper, we make use of the following notation: for a function $u$ defined on $\mathbb{R}^{3} \backslash \partial D$, we denote

$$
\left.u\right|_{ \pm}(\mathbf{x})=\lim _{\tau \rightarrow+0} u(\mathbf{x} \pm \tau \nu(\mathbf{x})), \quad \mathbf{x} \in \partial D
$$

and

$$
\left.\frac{\partial u}{\partial \nu}\right|_{ \pm}(\mathbf{x})=\lim _{\tau \rightarrow+0}\left\langle\nabla_{\mathbf{x}} u(\mathbf{x} \pm \tau \nu(\mathbf{x})), \nu(\mathbf{x})\right\rangle, \quad \mathbf{x} \in \partial D
$$

if the limits exist, where $\nu$ is the unit outward normal vector to $\partial D$.

It is known that the single layer potential $\mathcal{S}_{D}^{\omega}$ satisfies the trace formula (cf. (10,31)

$$
\left.\frac{\partial}{\partial \nu} \mathcal{S}_{D}^{\omega}[\varphi]\right|_{ \pm}=\left( \pm \frac{1}{2} I+\left(\mathcal{K}_{D}^{\omega}\right)^{*}\right)[\varphi] \quad \text { on } \partial D
$$

where $\left(\mathcal{K}_{D}^{\omega}\right)^{*}$ is the $L^{2}$-adjoint of $\mathcal{K}_{D}^{\omega}$ and

$$
\mathcal{K}_{D}^{\omega}[\mathbf{a}]:=\text { p.v. } \quad \int_{\partial D} \frac{\partial G_{\omega}(\mathbf{x}-\mathbf{y})}{\partial \nu(\mathbf{y})} \varphi(\mathbf{y}) d \sigma_{y}, \quad \mathbf{x} \in \partial D .
$$

The jump relations in the following proposition are also known (see [10,31]). 
Proposition 2.1. Let $\mathbf{a} \in \mathrm{TH}_{\mathrm{div}}^{-1 / 2}(\partial D)$. Then $\mathcal{A}_{D}^{\omega}[\mathbf{a}]$ is continuous on $\mathbb{R}^{3}$ and its curl satisfies the following jump formula,

$$
\nu \times \nabla \times\left.\mathcal{A}_{D}^{\omega}[\mathbf{a}]\right|_{ \pm}=\mp \frac{\mathbf{a}}{2}+\mathcal{M}_{D}^{\omega}[\mathbf{a}] \quad \text { on } \partial D
$$

where

$$
\nu(\mathbf{x}) \times \nabla \times\left.\mathcal{A}_{D}^{\omega}[\mathbf{a}]\right|_{ \pm}(\mathbf{x})=\lim _{t \rightarrow+0} \nu(\mathbf{x}) \times \nabla \times \mathcal{A}_{D}^{\omega}[\mathbf{a}](\mathbf{x} \pm t \nu(\mathbf{x})), \quad \forall \mathbf{x} \in \partial D,
$$

Equipped with the above knowledge, the solution pair $\left(\mathbf{E}_{\delta}, \mathbf{H}_{\delta}\right)$ in $\mathbb{R}^{3} \backslash \bar{D}_{\delta}$ to (1.14) can be represented using the following integral ansatz,

$$
\begin{array}{ll}
\mathbf{E}_{\delta}(\mathbf{x})=\mathbf{E}^{i}(\mathbf{x})+\nabla_{\mathbf{x}} \times \mathcal{A}_{D_{\delta}}^{\omega}[\mathbf{a}](\mathbf{x}), & \mathbf{x} \in \mathbb{R}^{3} \backslash \bar{D}_{\delta}, \\
\mathbf{H}_{\delta}(\mathbf{x})=\frac{1}{i \omega} \nabla_{\mathbf{x}} \times \mathbf{E}_{\delta}(\mathbf{x})=\mathbf{H}^{i}(\mathbf{x})+\frac{1}{i \omega} \nabla_{\mathbf{x}} \times \nabla_{\mathbf{x}} \times \mathcal{A}_{D_{\delta}}^{\omega}[\mathbf{a}](\mathbf{x}), & \mathbf{x} \in \mathbb{R}^{3} \backslash \bar{D}_{\delta},
\end{array}
$$

where by 2.6 the vectorial density function $\mathbf{a} \in \mathrm{TH}_{\mathrm{div}}^{-1 / 2}\left(\partial D_{\delta}\right)$ satisfies

$$
\left(-\frac{I}{2}+\mathcal{M}_{D_{\delta}}^{\omega}\right)[\mathbf{a}](\mathbf{x})=\nu \times\left.\left(\mathbf{E}_{\delta}-\mathbf{E}^{i}\right)(\mathbf{x})\right|_{+}, \quad \mathbf{y} \in \partial D_{\delta}
$$

\section{Regularized FULL-CLOAKING OF EM WAVES}

In this section, we consider a regularized full-cloaking scheme of the EM waves by taking the generating set to be a generic curve. As discussed in the introduction, this scheme was considered in our earlier work [9] for acoustic waves. For selfcontainedness, we briefly discuss the generating set $\Gamma_{0}$ and the virtual domain $D_{\delta}$ for the proposed cloaking scheme in the sequel, which can also be found in [9]. Let $\Gamma_{0}$ be a smooth simple and non-closed curve in $\mathbb{R}^{3}$ with two endpoints, denoted by $P_{0}$ and $Q_{0}$, respectively. Denote by $N(\mathbf{x})$ the normal plane of the curve $\Gamma_{0}$ at $\mathbf{x} \in \Gamma_{0}$. We note that $N\left(P_{0}\right)$ and $N\left(Q_{0}\right)$ are, respectively, defined by the left and right limits along $\Gamma_{0}$. Let $q \in \mathbb{R}_{+}$. For any $\mathbf{x} \in \Gamma_{0}$, we let $\mathscr{S}_{q}(\mathbf{x})$ denote the disk lying on $N(\mathbf{x})$, centered at $\mathbf{x}$ and of radius $q$. It is assumed that there exists $q_{0} \in \mathbb{R}_{+}$such that when $q \leq q_{0}, \mathscr{S}_{q}(\mathbf{x})$ intersects $\Gamma_{0}$ only at $\mathbf{x}$. We let $D_{q}^{f}$ be given as

$$
D_{q}^{f}:=\mathscr{S}_{q}(\mathbf{x}) \times \Gamma_{0}(\mathbf{x}), \mathbf{x} \in \bar{\Gamma}_{0},
$$

where $\Gamma_{0}$ is identified with its parametric representation $\Gamma_{0}(\mathbf{x})$; see Fig. 1 for a schematic illustration. Clearly, the facade of $D_{q}^{f}$, denoted by $S_{q}^{f}$ and parallel to $\Gamma_{0}$, is given by

$$
S_{q}^{f}:=\left\{\mathbf{x}+q \cdot \mathbf{n}(\mathbf{x}) ; \mathbf{x} \in \Gamma_{0}, \mathbf{n}(\mathbf{x}) \in N(\mathbf{x}) \cap \mathbb{S}^{2}\right\},
$$

and the two end-surfaces of $D_{q}^{f}$ are the two disks $\mathscr{S}_{q}\left(P_{0}\right)$ and $\mathscr{S}_{q}\left(Q_{0}\right)$. Let $D_{q_{0}}^{a}$ and $D_{q_{0}}^{b}$ be two simply connected sets with $\partial D_{q_{0}}^{a}=S_{q_{0}}^{a} \cup \mathscr{S}_{q_{0}}\left(P_{0}\right)$ and $\partial D_{q_{0}}^{b}=$ 


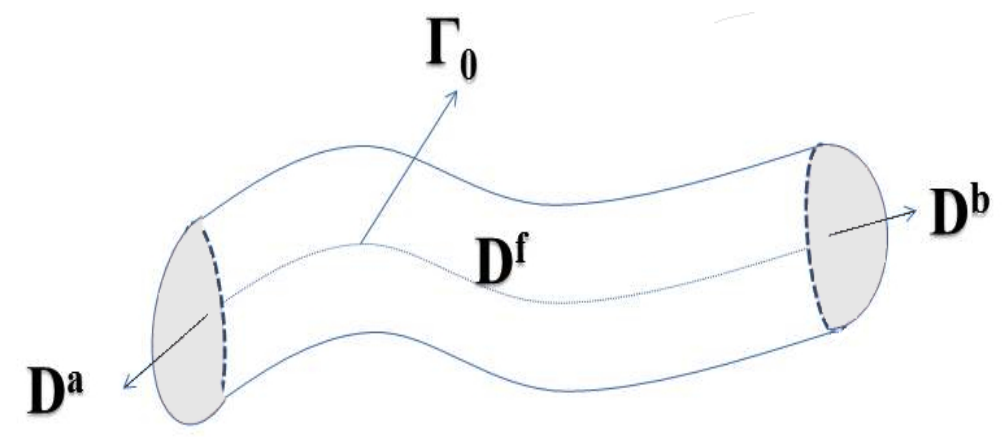

Figure 1. Schematic illustration of the domain $D_{q}$ for the regularized full-cloak.

$S_{q_{0}}^{b} \cup \mathscr{S}_{q_{0}}\left(Q_{0}\right)$, such that $S_{q_{0}}:=S_{q_{0}}^{f} \cup S_{q_{0}}^{b} \cup S_{q_{0}}^{a}$ is a $C^{3}$-smooth boundary of the domain $D_{q_{0}}:=D_{q_{0}}^{a} \cup D_{q_{0}}^{f} \cup D_{q_{0}}^{b}$. For $0<q<q_{0}$, we set

$$
D_{q}^{a}:=\frac{q}{q_{0}}\left(D_{q_{0}}^{a}-P_{0}\right)+P_{0}=\left\{\frac{q}{q_{0}} \cdot\left(\mathbf{x}-P_{0}\right)+P_{0} ; \mathbf{x} \in D_{q_{0}}^{a}\right\},
$$

and similarly, $D_{q}^{b}:=q / q_{0} \cdot\left(D_{q_{0}}^{b}-Q_{0}\right)+Q_{0}$. Let $S_{q}^{a}$ and $S_{q}^{b}$, respectively, denote the boundaries of $D_{q}^{a}$ and $D_{q}^{b}$ excluding $\mathscr{P}_{q}^{a}$ and $\mathscr{S}_{q}^{b}$. Now, we set $D_{q}:=D_{q}^{a} \cup D_{q}^{f} \cup D_{q}^{b}$, and $S_{q}:=S_{q}^{f} \cup S_{q}^{b} \cup S_{q}^{a}=\partial D_{q}$.

Henceforth, we we let $\delta \in \mathbb{R}_{+}$be the asymptotically small regularization parameter and let $D_{\delta}$ denote the virtual domain used for the blowup construction of the cloaking device. We also let

$$
S_{\delta}:=S_{\delta}^{f} \cup S_{\delta}^{b} \cup S_{\delta}^{a},
$$

denote the boundary surface of the virtual domain $D_{\delta}$. Without loss of generality, we assume that $q_{0} \equiv 1$. We shall drop the dependence on $q$ if one takes $q=1$. For example, $D$ and $S$ denote, respectively, $D_{q}$ and $S_{q}$ with $q=1$. It is remarked that in all of our subsequent arguments, $D$ can always be replaced by $D_{\tau_{0}}$ with $0<\tau_{0} \leq q_{0}$ being a fixed number.

In what follows, if we utilize $\mathbf{z}$ to denote the space variable on $\Gamma_{0}$, then for every $\mathbf{y} \in D_{q}^{f}$, we define a new variable $\mathbf{z}_{y} \in \Gamma_{0}$ which is the projection of $\mathbf{y}$ onto $\Gamma_{0}$. Meanwhile, if $\mathbf{y}$ belongs to $D_{q}^{a}$ (respectively $D_{q}^{b}$ ), then $\mathbf{z}_{y}$ is defined to be $P_{0}$ (respectively $Q_{0}$ ). Henceforth, we let $\xi$ denote the arc-length parameter of $\Gamma_{0}$ and $\theta$, which ranges from 0 to $2 \pi$, be the angle of the point on $N(\xi)$ with respect to the central point $\mathbf{x}(\xi) \in \Gamma_{0}$. Moreover, we assume that if $\theta=0$, then the corresponding points are those lying on the line that connects $\Gamma_{0}(\xi)$ to $\Gamma_{1}(\xi)$, where $\Gamma_{1}$ is defined to be

$$
\Gamma_{1}:=\left\{\mathbf{x}+\mathbf{n}_{1}(\mathbf{x}) ; \mathbf{x} \in \Gamma_{0}, \mathbf{n}_{1}(\mathbf{x}) \in N(\mathbf{x}) \cap \mathbb{S}^{2}-\text { a fixed vector for a given } \mathbf{x}\right\} .
$$


With the above preparation, we introduce a blowup transformation which maps $\mathbf{y} \in \bar{D}_{\delta}$ to $\tilde{\mathbf{y}} \in \bar{D}$ as follows

$$
A(\mathbf{y})=\tilde{\mathbf{y}}:=\frac{1}{\delta}\left(\mathbf{y}-\mathbf{z}_{y}\right)+\mathbf{z}_{y}, \quad \mathbf{y} \in D_{\delta}^{f},
$$

whereas

$$
A(\mathbf{y})=\tilde{\mathbf{y}}:= \begin{cases}\frac{\mathbf{y}-P_{0}}{\delta}+P_{0}, & \mathbf{y} \in D_{\delta}^{a}, \\ \frac{\mathbf{y}-Q_{0}}{\delta}+Q_{0}, & \mathbf{y} \in D_{\delta}^{b} .\end{cases}
$$

Next, we present the crucial design of the lossy layer in (1.3). Define the Jacobian matrix $\mathbf{B}$ by

$$
\mathbf{B}(\mathbf{y})=\nabla_{\mathbf{y}} A(\mathbf{y}), \quad \mathbf{y} \in \bar{D}_{\delta} .
$$

Set the material parameters $\varepsilon_{\delta}, \mu_{\delta}$ and $\sigma_{\delta}$ in the lossy layer $D_{\delta} \backslash \bar{D}_{\delta / 2}$ to be

$$
\begin{aligned}
& \varepsilon_{\delta}(\mathbf{x})=\varepsilon_{l}(\mathbf{x}):=\delta^{r}|\mathbf{B}| \mathbf{B}^{-1}, \quad \mu_{\delta}(\mathbf{x})=\mu_{l}(\mathbf{x}):=\delta^{s}|\mathbf{B}| \mathbf{B}^{-1}, \\
& \sigma_{\delta}(\mathbf{x})=\sigma_{l}(\mathbf{x}):=\delta^{t}|\mathbf{B}| \mathbf{B}^{-1},
\end{aligned}
$$

where $r, s$ and $t$ are all real numbers and $|\cdot|$ stands for the determinant when related to a square matrix.

We are now in a position to present the main theorem on the approximate full-cloak constructed by using $D_{\delta}$ described above as the virtual domain.

Theorem 3.1. Let $D_{\delta}$ be as described above with $\partial D_{\delta}=S_{\delta}$ defined in (3.3). Let $\left(\mathbf{E}_{\delta}, \mathbf{H}_{\delta}\right)$ be the pair of solutions to (1.14), with $\left\{\Omega ; \varepsilon_{\delta}, \mu_{\delta}, \sigma_{\delta}\right\} \subset\left\{\mathbb{R}^{3} ; \varepsilon_{\delta}, \mu_{\delta}, \sigma_{\delta}\right\}$ defined in (1.3), and $\left\{D_{\delta} \backslash \bar{D}_{\delta / 2} ; \varepsilon_{\delta}, \mu_{\delta}, \sigma_{\delta}\right\}$ given in (3.7). Define

$$
\beta=\min \{1,-1+r+s,-1+t+s\}, \quad \beta^{\prime}=\min \{1,-2+r+s,-2+t+s\} .
$$

If $r, s$ and $t$ are chosen such that $\beta^{\prime}-t / 2 \geq 0$, then there exists $\delta_{0} \in \mathbb{R}_{+}$such that when $\delta<\delta_{0}$,

$$
\left\|\mathbf{A}_{\infty}^{\delta}(\hat{\mathbf{x}} ; \mathbf{p}, \mathbf{d})\right\| \leq C\left(\delta^{\beta-t / 2+1}+\delta^{2}\right)
$$

where $C$ is a positive constant depending on $\omega$ and $D$, but independent of $\varepsilon_{a}, \mu_{a}$, $\sigma_{a}$ and $\hat{\mathbf{x}}, \mathbf{p}, \mathbf{d}$.

Remark 3.1. Following our earlier discussion, one can immediately infer by Theorems 1.1 and 3.1 that the push-forwarded structure in 1.2 ,

$$
\{\Omega ; \varepsilon, \mu, \sigma\}=\left(F_{\delta}\right)_{*}\left\{\Omega ; \varepsilon_{\delta}, \mu_{\delta}, \sigma_{\delta}\right\}
$$

produces an approximate full-cloaking device within at least $\delta$-accuracy to the ideal cloak. Indeed, if we set $s=2$ and $r=t=0$ then one has $\beta=1, \beta^{\prime}=0$ and the accuracy of the ideal cloak will be $\delta^{2}$, which is the highest accuracy that one can obtain for such a construction. Particularly, it is emphasize that in (3.8), the estimate is independent of $\varepsilon_{a}, \mu_{a}, \sigma_{a}$, and this means that the cloaked content $\left\{\Omega_{a} ; \varepsilon_{a}^{*}, \mu_{a}^{*}, \sigma_{a}^{*}\right\}$ in $(1.2)$ can be arbitrary but regular. Finally, as remarked earlier, we refer to 25 for the construction of the blowup transformation $F_{\delta}$, which we always assume the existence in the current study. 
The subsequent three subsections are devoted to the proof of Theorem 3.1. For our later use, we first derive some critical lemmas.

3.1. Auxiliary lemmas. In this subsection we present some auxiliary lemmas that are essential for our analysis of the far field estimates. To begin with, we show the following properties of the blowup transformation defined in (3.4)

Lemma 3.1 (Lemma 4.1 in [9]). Let $A$ be the transformation introduced in (3.4) and (3.5) which maps the region $\bar{D}_{\delta}$ to $\bar{D}$. Let $\mathbf{B}(\mathbf{y})$ be the corresponding Jacobian matrix of $A(\mathbf{y})$ given by (3.6). Then we have

$$
\mathbf{B}(\mathbf{y})= \begin{cases}\frac{1}{\delta} \mathbf{I}_{3 \times 3}-\left(\frac{1}{\delta}-1\right) \mathbf{z}_{y}^{\prime}(\xi) \mathbf{z}_{y}^{\prime}(\xi)^{T}, & \mathbf{y} \in D_{\delta}^{f}, \\ \frac{1}{\delta} \mathbf{I}_{3 \times 3}, & \mathbf{y} \in D_{\delta}^{a} \cup D_{\delta}^{b},\end{cases}
$$

where the superscript $T$ denotes the transpose of a vector or a matrix. Furthermore,

$$
\mathbf{B}(\mathbf{y}) \nu_{\mathbf{y}}=\frac{1}{\delta} \nu_{\mathbf{y}}, \quad \mathbf{y} \in \partial D_{\delta},
$$

where $\nu_{\mathbf{y}}$ stands for the unit outward normal vector to $\partial D_{\delta}$ at $\mathbf{y} \in \partial D_{\delta}$.

Remark 3.2. In view of the Jacobian matrix form 3.9 , one can also find that the eigenvalues of $\mathbf{B}(\mathbf{x}), \mathbf{x} \in D_{\delta}$ are either 1 or $1 / \delta$. Hence for any vector field $\mathbf{V} \in \mathbb{R}^{3}$, there holds

$$
\|\mathbf{V}\|^{2} \leq\langle\mathbf{B}(\mathbf{x}) \mathbf{V}, \mathbf{V}\rangle \leq \delta^{-1}\|\mathbf{V}\|^{2}
$$

uniformly for $\mathbf{x} \in D_{\delta}$. It can also be easily seen from $(3.9)$ that

$$
|\mathbf{B}(\mathbf{y})|=\delta^{-2}, \quad \mathbf{y} \in D_{\delta}^{f} .
$$

For the sake of simplicity, we define

$$
\mathbf{E}_{\delta}^{+}:=\mathbf{E}_{\delta}-\mathbf{E}^{i}, \quad \mathbf{H}_{\delta}^{+}:=\mathbf{H}_{\delta}-\mathbf{H}^{i}, \quad \text { in } \quad \mathbb{R}^{3} \backslash \bar{D}_{\delta} .
$$

Furthermore, we introduce the following notations

$$
\tilde{\mathbf{E}}(\tilde{\mathbf{x}}):=\mathbf{E}\left(A^{-1}(\tilde{\mathbf{x}})\right)=\mathbf{E}(\mathbf{x}), \quad \tilde{\mathbf{H}}(\tilde{\mathbf{x}}):=\mathbf{H}\left(A^{-1}(\tilde{\mathbf{x}})\right)=\mathbf{H}(\mathbf{x}),
$$

and define the corresponding fields after change of variables by

$$
\hat{\mathbf{E}}(\tilde{\mathbf{x}}):=\left(\left(\tilde{\mathbf{B}}^{T}\right)^{-1} \tilde{\mathbf{E}}\right)(\tilde{\mathbf{x}}), \quad \hat{\mathbf{H}}(\tilde{\mathbf{x}}):=\left(\left(\tilde{\mathbf{B}}^{T}\right)^{-1} \tilde{\mathbf{H}}\right)(\tilde{\mathbf{x}}),
$$

where

$$
\tilde{\mathbf{B}}(\tilde{\mathbf{x}}):=\mathbf{B}\left(A^{-1}(\tilde{\mathbf{x}})\right)=\mathbf{B}(\mathbf{x}) .
$$

We mention that sometimes we write $\mathbf{B}$ and $\tilde{\mathbf{B}}$ in the sequel and omit their dependences for simplicity. The following lemma is of critical importance for our subsequent analysis. 
Lemma 3.2 (Corollary 3.58 in [32]). Let $\tilde{\mathbf{x}}=A(\mathbf{x})$ with $\mathbf{B}(\mathbf{x})=\nabla A(\mathbf{x})$. Then for the bounded domain $D_{\delta}$ and any vector field $\mathbf{V} \in H\left(\operatorname{curl} ; D_{\delta}\right)$,

$$
\hat{\mathbf{V}}(\tilde{\mathbf{x}}):=\left(\tilde{\mathbf{B}}^{T}\right)^{-1} \tilde{\mathbf{V}}(\tilde{\mathbf{x}}), \quad \tilde{\mathbf{V}}(\tilde{\mathbf{x}}):=\mathbf{V}(\mathbf{x}), \quad \mathbf{x} \in D_{\delta}
$$

there hold the following identities

$$
|\tilde{\mathbf{B}}|^{-1} \tilde{\mathbf{B}}(\nabla \times \mathbf{V})\left(A^{-1}(\tilde{\mathbf{x}})\right)=\nabla_{\tilde{\mathbf{x}}} \times \hat{\mathbf{V}}(\tilde{\mathbf{x}})
$$

and

$$
\int_{\partial D_{\delta}}\left(\nu_{\mathbf{x}} \times \mathbf{V}\right) \cdot \mathbf{W} d \sigma_{x}=\int_{\partial D}\left(\nu_{\tilde{\mathbf{x}}} \times \hat{\mathbf{V}}\right) \cdot \hat{\mathbf{W}} d \sigma_{\tilde{x}}
$$

where $\mathbf{W} \in H\left(\operatorname{curl} ; D_{\delta}\right)$ and $\hat{\mathbf{W}}(\tilde{\mathbf{x}}):=\left(\tilde{\mathbf{B}}^{T}\right)^{-1} \tilde{\mathbf{W}}(\tilde{\mathbf{x}}):=\left(\mathbf{B}^{T}\right)^{-1} \mathbf{W}(\mathbf{x})$.

Note that

$$
d \sigma_{y}= \begin{cases}\delta d \sigma_{\tilde{y}}, & \mathbf{y} \in S_{\delta}^{f} \\ \delta^{2} d \sigma_{\tilde{y}}, & \mathbf{y} \in S_{\delta}^{b} \cup S_{\delta}^{a},\end{cases}
$$

with which one can show that

Lemma 3.3. Let $D^{c}, c \in\{f, a, b\}$ be defined at the beginning of this section. Let $\mathbf{V}$ and $\hat{\mathbf{V}}$, B be similarly defined as those in Lemma 3.2. Then for any $\mathbf{W} \in$ $H(\operatorname{curl} ; D)$, one has

$$
\begin{aligned}
& \int_{\partial D^{f}} \nu_{\tilde{\mathbf{x}}} \times \tilde{\mathbf{V}} \cdot \mathbf{W}(\tilde{\mathbf{x}}) d \sigma_{\tilde{x}}=\delta^{-1} \int_{\partial D^{f}} \tilde{\nu}_{\tilde{\mathbf{x}}} \times \hat{\mathbf{V}} \cdot \hat{\mathbf{W}}(\tilde{\mathbf{x}}) d \sigma_{\tilde{x}} \\
& \int_{\partial D^{c}} \nu_{\tilde{\mathbf{x}}} \times \tilde{\mathbf{V}} \cdot \mathbf{W}(\tilde{\mathbf{x}}) d \sigma_{\tilde{x}}=\delta^{-2} \int_{\partial D^{c}} \nu_{\tilde{\mathbf{x}}} \times \hat{\mathbf{V}} \cdot \hat{\mathbf{W}}(\tilde{\mathbf{x}}) d \sigma_{\tilde{x}} \quad c \in\{a, b\},
\end{aligned}
$$

where $\hat{\mathbf{W}}(\tilde{\mathbf{x}}):=\left(\tilde{\mathbf{B}}^{T}\right)^{-1} \mathbf{W}(\tilde{\mathbf{x}})$.

Proof. The proof follows directly from (3.17), 3.18) by using change of variables in the corresponding integrals.

Lemma 3.4. Suppose $\tilde{\mathbf{a}}(\tilde{\mathbf{x}})=\mathbf{a}(\mathbf{x})$ for $\mathbf{x} \in \partial D_{\delta}$ and $\tilde{\mathbf{x}} \in \partial D$. Define

$$
\mathcal{M}_{S^{f}}^{\omega}[\tilde{\mathbf{a}}](\tilde{\mathbf{x}}):=\text { p.v. } \quad \nu_{\tilde{\mathbf{x}}} \times \nabla_{\mathbf{z}_{\tilde{x}}} \times \int_{S^{f}} G_{\omega}\left(\mathbf{z}_{\tilde{x}}-\mathbf{z}_{\tilde{y}}\right) \tilde{\mathbf{a}}(\tilde{\mathbf{y}}) d \sigma_{\tilde{y}},
$$

and

Define

$$
\mathcal{M}_{S^{c}}[\tilde{\mathbf{a}}](\tilde{\mathbf{x}}):=\text { p.v. } \quad \nu_{\tilde{\mathbf{x}}} \times \nabla_{\tilde{\mathbf{x}}} \times \int_{S^{c}} G_{\omega}(\tilde{\mathbf{x}}-\tilde{\mathbf{y}}) \tilde{\mathbf{a}}(\tilde{\mathbf{y}}) d \sigma_{\tilde{y}}
$$

$$
\mathcal{L}_{D_{\delta}}^{\omega}(\mathbf{x}):=\nu_{\mathbf{x}} \times \nabla_{\mathbf{x}} \times \nabla_{\mathbf{x}} \times \mathcal{A}_{D_{\delta}}^{\omega}[\mathbf{a}](\mathbf{x}) .
$$

Then there hold the following results

$$
\mathcal{M}_{D_{\delta}}^{\omega}[\mathbf{a}](\mathbf{x})= \begin{cases}\delta \mathcal{M}_{S^{f}}^{\omega}[\tilde{\mathbf{a}}](\tilde{\mathbf{x}})+\mathcal{O}\left(\delta^{2}\|\tilde{\mathbf{a}}\|_{\mathrm{TH}^{-1 / 2}(\partial D)}\right), & \mathbf{x} \in S_{\delta}^{f}, \\ \mathcal{M}_{S^{c}}[\tilde{\mathbf{a}}](\tilde{\mathbf{x}})+\mathcal{O}\left(\delta\|\tilde{\mathbf{a}}\|_{\mathrm{TH}^{-1 / 2}(\partial D)}\right), & \mathbf{x} \in S_{\delta}^{c},\end{cases}
$$


and

$$
\mathcal{L}_{D_{\delta}}^{\omega}[\mathbf{a}](\mathbf{x})= \begin{cases}\delta \mathcal{L}_{S^{f}}^{\omega}[\tilde{\mathbf{a}}](\tilde{\mathbf{x}})+\mathcal{O}\left(\delta^{2}\|\tilde{\mathbf{a}}\|_{\mathrm{TH}^{-1 / 2}(\partial D)}\right), & \mathbf{x} \in S_{\delta}^{f}, \\ \frac{1}{\delta} \nu_{\tilde{\mathbf{x}}} \times \nabla_{\tilde{\mathbf{x}}} \times \nabla_{\tilde{\mathbf{x}}} \times \mathcal{A}_{S^{c}}[\tilde{\mathbf{a}}](\tilde{\mathbf{x}})+\mathcal{O}\left(\delta\|\tilde{\mathbf{a}}\|_{\mathrm{TH}^{-1 / 2}(\partial D)}\right), & \mathbf{x} \in S_{\delta}^{c}\end{cases}
$$

for $c \in\{a, b\}$, where

$$
\mathcal{L}_{S^{f}}^{\omega}[\tilde{\mathbf{a}}](\mathbf{x}):=\nu_{\tilde{\mathbf{x}}} \times \nabla_{\mathbf{z}_{\tilde{x}}} \times \nabla_{\mathbf{z}_{\tilde{x}}} \times \int_{S^{f}} G_{\omega}\left(\mathbf{z}_{\tilde{x}}-\mathbf{z}_{\tilde{y}}\right) \tilde{\mathbf{a}}(\tilde{\mathbf{y}}) d \sigma_{\tilde{y}},
$$

and $\mathcal{A}_{S^{c}}$ is given in 2.2 by replacing $\partial D$ and $\omega$ with $S^{c}$ and 0 , respectively.

Proof. For $\mathbf{x}, \mathbf{y} \in D_{\delta}$, one has

$$
\mathbf{x}-\mathbf{y}=\left(\mathbf{x}-\mathbf{z}_{x}\right)-\left(\mathbf{y}-\mathbf{z}_{y}\right)+\mathbf{z}_{x}-\mathbf{z}_{y}=\delta\left(\left(\tilde{\mathbf{x}}-\mathbf{z}_{\tilde{x}}\right)-\left(\tilde{\mathbf{y}}-\mathbf{z}_{\tilde{y}}\right)\right)+\left(\mathbf{z}_{\tilde{x}}-\mathbf{z}_{\tilde{y}}\right) .
$$

Hence, we have the following expansion for $\mathbf{x} \in S_{\delta}^{f}$,

$$
\|\mathbf{x}-\mathbf{y}\|=\left\|\mathbf{z}_{\tilde{x}}-\mathbf{z}_{\tilde{y}}\right\|+\delta\left\langle\left(\tilde{\mathbf{x}}-\mathbf{z}_{\tilde{x}}\right)-\left(\tilde{\mathbf{y}}-\mathbf{z}_{\tilde{y}}\right), \frac{\mathbf{z}_{\tilde{x}}-\mathbf{z}_{\tilde{y}}}{\left\|\mathbf{z}_{\tilde{x}}-\mathbf{z}_{\tilde{y}}\right\|}\right\rangle+\mathcal{O}\left(\delta^{2}\right) .
$$

Similarly

$$
\begin{aligned}
& \left\langle\mathbf{x}-\mathbf{y}, \nu_{\mathbf{x}}\right\rangle=\left\langle\mathbf{z}_{\tilde{x}}-\mathbf{z}_{\tilde{y}}, \nu_{\mathbf{x}}\right\rangle+\delta\left\langle\left(\tilde{\mathbf{x}}-\mathbf{z}_{\tilde{x}}\right)-\left(\tilde{\mathbf{y}}-\mathbf{z}_{\tilde{y}}\right), \nu_{\mathbf{x}}\right\rangle, \\
& \|\mathbf{x}-\mathbf{y}\|^{-1}=\left\|\mathbf{z}_{\tilde{x}}-\mathbf{z}_{\tilde{y}}\right\|^{-1}-\delta\left\langle\left(\tilde{\mathbf{x}}-\mathbf{z}_{\tilde{x}}\right)-\left(\tilde{\mathbf{y}}-\mathbf{z}_{\tilde{y}}\right), \frac{\mathbf{z}_{\tilde{x}}-\mathbf{z}_{\tilde{y}}}{\left\|\mathbf{z}_{\tilde{x}}-\mathbf{z}_{\tilde{y}}\right\|^{3}}\right\rangle+\mathcal{O}\left(\delta^{2}\right), \\
& e^{i \omega\|\mathbf{x}-\mathbf{y}\|}=e^{i \omega\left\|\mathbf{z}_{\tilde{x}}-\mathbf{z}_{\tilde{y}}\right\|}\left(1+i \omega \delta\left\langle\left(\tilde{\mathbf{x}}-\mathbf{z}_{\tilde{x}}\right)-\left(\tilde{\mathbf{y}}-\mathbf{z}_{\tilde{y}}\right), \frac{\mathbf{z}_{\tilde{x}}-\mathbf{z}_{\tilde{y}}}{\left\|\mathbf{z}_{\tilde{x}}-\mathbf{z}_{\tilde{y}}\right\|}\right)+\mathcal{O}\left(\delta^{2}\right) .\right.
\end{aligned}
$$

With those expansions at hand, we proceed to compute for $\mathbf{x} \in S_{\delta}^{f}$,

$$
\begin{aligned}
\nabla_{\mathbf{x}} G_{\omega}(\mathbf{x}-\mathbf{y}) & =\frac{(\mathbf{x}-\mathbf{y}) e^{i \omega\|\mathbf{x}-\mathbf{y}\|}}{4 \pi\|\mathbf{x}-\mathbf{y}\|^{2}}\left(\frac{1}{\|\mathbf{x}-\mathbf{y}\|}-i \omega\right) \\
& =\frac{\left(\mathbf{z}_{\tilde{x}}-\mathbf{z}_{\tilde{y}}\right) e^{i \omega\left\|\mathbf{z}_{\tilde{x}}-\mathbf{z}_{\tilde{y}}\right\|}}{4 \pi\left\|\mathbf{z}_{\tilde{x}}-\mathbf{z}_{\tilde{y}}\right\|^{2}}\left(\frac{1}{\left\|\mathbf{z}_{\tilde{x}}-\mathbf{z}_{\tilde{y}}\right\|}-i \omega\right)+\mathcal{O}(\delta) \\
& =\nabla_{\mathbf{z}_{\tilde{x}}} G_{\omega}\left(\mathbf{z}_{\tilde{x}}-\mathbf{z}_{\tilde{y}}\right)+\mathcal{O}(\delta),
\end{aligned}
$$

and by using vector calculus identities,

$$
\begin{aligned}
& \mathcal{M}_{D_{\delta}}^{\omega}[\mathbf{a}](\mathbf{x})=\nu_{\mathbf{x}} \times \nabla_{\mathbf{x}} \times \int_{\partial D_{\delta}} G_{\omega}(\mathbf{x}-\mathbf{y}) \mathbf{a}(\mathbf{y}) d \sigma_{y} \\
= & \int_{\partial D_{\delta}} \nabla_{\mathbf{x}} G_{\omega}(\mathbf{x}-\mathbf{y}) \nu_{\mathbf{x}} \cdot \mathbf{a}(\mathbf{y}) d \sigma_{y}-\int_{\partial D_{\delta}} \nu_{\mathbf{x}} \cdot \nabla_{\mathbf{x}} G_{\omega}(\mathbf{x}-\mathbf{y}) \mathbf{a}(\mathbf{y}) d \sigma_{y} \\
= & \delta \int_{S^{f}} \nabla_{\mathbf{z}_{\tilde{x}}} G_{\omega}\left(\mathbf{z}_{\tilde{x}}-\mathbf{z}_{\tilde{y}}\right) \nu_{\tilde{\mathbf{x}}} \cdot \tilde{\mathbf{a}}(\tilde{\mathbf{y}}) d \sigma_{\tilde{y}}-\delta \int_{S^{f}} \nu_{\tilde{\mathbf{x}}} \cdot \nabla_{\mathbf{z}_{\tilde{x}}} G_{\omega}\left(\mathbf{z}_{\tilde{x}}-\mathbf{z}_{\tilde{y}}\right) \tilde{\mathbf{a}}(\tilde{\mathbf{y}}) d \sigma_{\tilde{y}} \\
& +\mathcal{O}\left(\delta^{2}\|\tilde{\mathbf{a}}\|_{\mathrm{TH}^{-1 / 2}(\partial D)}\right)=\delta \mathcal{M}_{S^{f}}^{\omega}[\tilde{\mathbf{a}}](\tilde{\mathbf{x}})+\mathcal{O}\left(\delta^{2}\|\tilde{\mathbf{a}}\|_{\mathrm{TH}^{-1 / 2}(\partial D)}\right),
\end{aligned}
$$


which proves the case of 3.21 for $\mathbf{x} \in S_{\delta}^{f}$. Next, note that if $\mathbf{x} \in S_{\delta}^{c}, c \in\{a, b\}$, then

$$
\mathbf{z}_{\tilde{x}}-\mathbf{z}_{\tilde{y}}=0 \quad \text { for } \quad \mathbf{y} \in S_{\delta}^{c}
$$

and

$$
\|\mathbf{x}-\mathbf{y}\|=\delta\|\tilde{\mathbf{x}}-\tilde{\mathbf{y}}\|, \quad \frac{\left\langle\mathbf{x}-\mathbf{y}, \nu_{\mathbf{x}}\right\rangle}{\|\mathbf{x}-\mathbf{y}\|}=\frac{\left\langle\tilde{\mathbf{x}}-\tilde{\mathbf{y}}, \nu_{\mathbf{x}}\right\rangle}{\|\tilde{\mathbf{x}}-\tilde{\mathbf{y}}\|}, \quad \mathbf{x}, \mathbf{y} \in S_{\delta}^{c} .
$$

With the above facts and by using a similar argument to the proof of the first case of (3.21), one can prove (3.21) for $\mathbf{x} \in S_{\delta}^{c}, c \in\{a, b\}$. To prove (3.22), we first note that

$$
\nabla_{\mathbf{x}} \times \nabla_{\mathbf{x}} \times \mathcal{A}_{D_{\delta}}^{\omega}[\mathbf{a}](\mathbf{x})=\omega^{2} \mathcal{A}_{D_{\delta}}^{\omega}[\mathbf{a}](\mathbf{x})+\nabla_{\mathbf{x}} \nabla_{\mathbf{x}} \cdot \mathcal{A}_{D_{\delta}}^{\omega}[\mathbf{a}](\mathbf{x})
$$

By using (4.17) in [9], one can easily obtain

$$
\mathcal{A}_{D_{\delta}}^{\omega}[\mathbf{a}](\mathbf{x})= \begin{cases}\delta \mathcal{A}_{S^{f}}^{\omega}[\tilde{\mathbf{a}}](\tilde{\mathbf{x}})+\mathcal{O}\left(\delta^{2}\|\tilde{\mathbf{a}}\|_{\mathrm{TH}^{-1 / 2}(\partial D)}\right), & \mathbf{x} \in S_{\delta}^{f}, \\ \delta\left(\mathcal{A}_{S^{c}}+\mathcal{A}_{S^{f}}^{\omega}\right)[\tilde{\mathbf{a}}](\tilde{\mathbf{x}})+\mathcal{O}\left(\delta^{2}\|\tilde{\mathbf{a}}\|_{\mathrm{TH}^{-1 / 2}(\partial D)}\right), & \mathbf{x} \in S_{\delta}^{c},\end{cases}
$$

for $c \in\{a, b\}$, where $\mathcal{A}_{S^{f}}^{\omega}$ is defined similarly as $\mathcal{S}_{S^{f}}^{\omega}$ in $[9]$. Using similar expansion strategy, one can prove for $\mathrm{x} \in S_{\delta}^{f}$

$$
\nabla_{\mathbf{x}} \nabla_{\mathbf{x}} \cdot \mathcal{A}_{D_{\delta}}^{\omega}[\mathbf{a}](\mathbf{x})=\delta \nabla_{\mathbf{z}_{x}} \nabla_{\mathbf{z}_{x}} \cdot \mathcal{A}_{S^{f}}^{\omega}[\tilde{\mathbf{a}}](\tilde{\mathbf{x}})+\mathcal{O}\left(\delta^{2}\|\tilde{\mathbf{a}}\|_{\mathrm{TH}^{-1 / 2}(\partial D)}\right)
$$

Note that for $\mathbf{x} \in S_{\delta}^{c}, c \in\{a, b\}$, there holds (see Proposition 5.2 in [2] for details)

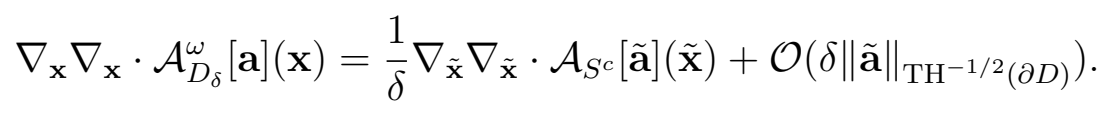

By combining (3.23)-3.26) together, one can complete the proof.

3.2. Asymptotic expansions. In order to tackle the integral equation (2.9), we shall first derive some crucial asymptotic expansions. Henceforth, we denote $\tilde{\mathbf{a}}(\tilde{\mathbf{y}}):=\mathbf{a}(\mathbf{y})$ for $\tilde{\mathbf{y}}=A(\mathbf{y}), \mathbf{y} \in D_{\delta}$ and $\tilde{\mathbf{y}} \in \partial D$. The same notation shall be adopted for $\mathbf{x}$ and $\tilde{\mathbf{x}}$.

For $\mathbf{x} \in \mathbb{R}^{3} \backslash D_{\delta}$ with sufficiently large $\|\mathbf{x}\|$, we can expand $\mathbf{E}_{\delta}-\mathbf{E}^{i}$ from 2.7) in $\mathbf{z} \in \Gamma_{0}$ as follows

$$
\begin{aligned}
& \left(\mathbf{E}_{\delta}-\mathbf{E}^{i}\right)(\mathbf{x})=\nabla \times \mathcal{A}_{D_{\delta}}^{\omega}[\mathbf{a}](\mathbf{x})=\nabla \times \int_{\partial D_{\delta}} G_{\omega}(\mathbf{x}-\mathbf{y}) \mathbf{a}(\mathbf{y}) d \sigma_{y} \\
= & \nabla \times \int_{\partial D_{\delta}} G_{\omega}\left(\mathbf{x}-\mathbf{z}_{y}\right) \mathbf{a}(\mathbf{y}) d \sigma_{y}-\nabla \times \int_{\partial D_{\delta}} \nabla G_{\omega}\left(\mathbf{x}-\mathbf{z}_{y}\right) \cdot\left(\mathbf{y}-\mathbf{z}_{y}\right) \mathbf{a}(\mathbf{y}) d \sigma_{y} \\
& +\nabla \times \int_{\partial D_{\delta}}\left(\mathbf{y}-\mathbf{z}_{y}\right)^{T} \nabla^{2} G_{\omega}(\mathbf{x}-\zeta(\mathbf{y}))\left(\mathbf{y}-\mathbf{z}_{y}\right) \mathbf{a}(\mathbf{y}) d \sigma_{y} \\
:= & R_{1}+R_{2}+R_{3},
\end{aligned}
$$

where $\zeta(\mathbf{y})=\eta \mathbf{y}+(1-\eta) \mathbf{z}_{y} \in D_{\delta}$ for some $\eta \in(0,1)$, and the superscript $T$ signifies the matrix transpose. We next estimate the three terms $R_{1}, R_{2}$ and $R_{3}$ in 
(3.27). The term $R_{3}$ in (3.27) is a remainder term from the Taylor series expansion and it verifies the following estimate,

$$
\begin{aligned}
& \left\|R_{3}\right\|_{L^{\infty}\left(\mathbb{S}^{2}\right)^{3}}=\delta^{2}\left\|\nabla \times \int_{\partial D_{\delta}}\left(\tilde{\mathbf{y}}-\mathbf{z}_{\tilde{y}}\right)^{T} \nabla^{2} G_{\omega}(\mathbf{x}-\zeta(\mathbf{y}))\left(\tilde{\mathbf{y}}-\mathbf{z}_{\tilde{y}}\right) \mathbf{a}(\mathbf{y}) d \sigma_{y}\right\|_{L^{\infty}\left(\mathbb{S}^{2}\right)^{3}} \\
\leq & C \delta^{3} \frac{1}{\|\mathbf{x}\|}\|\tilde{\mathbf{a}}\|_{\mathrm{TH}^{-1 / 2}(\partial D)} .
\end{aligned}
$$

In the sequel, we shall need the expansion of the incident plane wave $\mathbf{E}^{i}$ in $\mathbf{z} \in \Gamma_{0}$, and there holds

$$
\mathbf{E}^{i}(\mathbf{y})=\mathbf{E}^{i}\left(\mathbf{z}_{y}\right)+\nabla \mathbf{E}^{i}\left(\mathbf{z}_{y}\right) \cdot\left(\mathbf{y}-\mathbf{z}_{y}\right)+\sum_{|\alpha|=2}^{\infty} \partial_{\mathbf{y}}^{\alpha} \mathbf{E}^{i}\left(\mathbf{z}_{y}\right)\left(\mathbf{y}-\mathbf{z}_{y}\right)^{\alpha},
$$

where the multi-index $\alpha=\left(\alpha_{1}, \alpha_{2}, \alpha_{3}\right)$ and $\partial_{\mathbf{y}}^{\alpha}=\partial_{y_{1}}^{\alpha_{1}} \partial_{y_{2}}^{\alpha_{2}} \partial_{y_{3}}^{\alpha_{3}}$ with $\mathbf{y}=\left(y_{1}, y_{2}, y_{3}\right)$. Since for $\mathbf{y} \in \partial D_{\delta}, \nu_{\mathbf{y}}=\nu_{\tilde{\mathbf{y}}}$, one further has

$$
\nu_{\mathbf{y}} \times \mathbf{E}^{i}(\mathbf{y})=\nu_{\tilde{\mathbf{y}}} \times \sum_{|\alpha|=0}^{\infty} \delta^{\alpha} \partial_{\mathbf{y}}^{\alpha} \mathbf{E}^{i}\left(\mathbf{z}_{y}\right)\left(\tilde{\mathbf{y}}-\mathbf{z}_{\tilde{y}}\right)^{\alpha} .
$$

In what follows, we define

$$
\tilde{\Phi}(\tilde{\mathbf{y}}):=\Phi(\mathbf{y})=\nu_{\mathbf{y}} \times\left.\mathbf{E}_{\delta}(\mathbf{y})\right|_{\partial D_{\delta}} ^{+} .
$$

Theorem 3.2. Let $\mathbf{E}_{\delta}$ be the solution to 1.14), then there holds for $\mathbf{x} \in \mathbb{R}^{3} \backslash \bar{D}$,

$$
\int_{S^{f}} G_{\omega}\left(\mathbf{x}-\mathbf{z}_{\tilde{y}}\right) \tilde{\mathbf{a}}(\tilde{\mathbf{y}}) d \sigma_{\tilde{y}}=-2 \int_{S^{f}} G_{\omega}\left(\mathbf{x}-\mathbf{z}_{\tilde{y}}\right) \tilde{\Phi}(\tilde{\mathbf{y}}) d \sigma_{\tilde{y}}+\mathcal{O}\left(\delta\left(\|\tilde{\Phi}\|_{\mathrm{TH}^{-1 / 2}(\partial D)}+1\right)\right) .
$$

If one assumes that $\Phi(\mathbf{y})=0, \mathbf{y} \in \partial D_{\delta}$, then there holds for $\mathbf{x} \in \mathbb{R}^{3} \backslash \bar{D}$,

$$
\begin{aligned}
\left(\mathbf{E}_{\delta}-\mathbf{E}^{i}\right)(\mathbf{x})=2 \delta^{2} \nabla & \times\left(\int_{S^{f}} G_{\omega}\left(\mathbf{x}-\mathbf{z}_{\tilde{y}}\right) \nu_{\tilde{\mathbf{y}}} \times\left(\nabla \mathbf{E}^{i}\left(\mathbf{z}_{\tilde{y}}\right)\left(\tilde{\mathbf{y}}-\mathbf{z}_{\tilde{y}}\right)\right) d \sigma_{y}\right. \\
& +\int_{S^{a} \cup S^{b}} G_{\omega}\left(\mathbf{x}-\mathbf{z}_{\tilde{y}}\right) \nu_{\tilde{\mathbf{y}}} \times \mathbf{E}^{i}\left(\mathbf{z}_{\tilde{y}}\right) d \sigma_{\tilde{y}} \\
& \left.-\int_{S^{f}}\left(\nabla G_{\omega}\left(\mathbf{x}-\mathbf{z}_{\tilde{y}}\right) \cdot\left(\tilde{\mathbf{y}}-\mathbf{z}_{\tilde{y}}\right)\right) \nu_{\tilde{\mathbf{y}}} \times \mathbf{E}^{i}\left(\mathbf{z}_{\tilde{y}}\right)(\tilde{\mathbf{y}}) d \sigma_{\tilde{y}}\right)+\mathcal{O}\left(\delta^{3}\right) .
\end{aligned}
$$

Proof. Recall that $-\frac{I}{2}+\mathcal{M}_{D_{\delta}}^{\omega}$ is invertible on $\mathrm{TH}_{\mathrm{div}}^{-1 / 2}\left(\partial D_{\delta}\right)$ (see, e.g. 18]). By (2.9), we see that

$$
\mathbf{a}(\mathbf{x})=\left(-\frac{I}{2}+\mathcal{M}_{D_{\delta}}^{\omega}\right)^{-1}\left[\nu_{\mathbf{y}} \times\left.\left(\mathbf{E}_{\delta}-\mathbf{E}^{i}\right)(\mathbf{y})\right|_{\partial D_{\delta}} ^{+}\right](\mathbf{x})
$$


Using the results in Lemma 3.4 and the expansion of $\mathbf{E}^{i}$ in 3.29 , we have for $\mathbf{x} \in S_{\delta}^{f}$ that

$$
\begin{aligned}
\tilde{\mathbf{a}}(\tilde{\mathbf{x}})= & -2 \tilde{\Phi}(\tilde{\mathbf{x}})+2 \nu_{\tilde{\mathbf{x}}} \times \mathbf{E}^{i}\left(\mathbf{z}_{\tilde{x}}\right)-4 \delta \mathcal{M}_{S^{f}}^{\omega}[\tilde{\Phi}](\tilde{\mathbf{x}})+2 \delta \nu_{\tilde{\mathbf{x}}} \times\left(\nabla \mathbf{E}^{i}\left(\mathbf{z}_{\tilde{x}}\right)\left(\tilde{\mathbf{x}}-\mathbf{z}_{\tilde{x}}\right)\right) \\
& +4 \delta \mathcal{M}_{S^{f}}^{\omega}\left[\nu_{\tilde{\mathbf{y}}} \times \mathbf{E}^{i}\left(\mathbf{z}_{\tilde{y}}\right)\right](\tilde{\mathbf{x}})+\mathcal{O}\left(\delta^{2}\left(\|\tilde{\Phi}\|_{\mathrm{TH}^{-1 / 2}(\partial D)}+1\right)\right) .
\end{aligned}
$$

Note that

$$
\int_{S^{f}} G_{\omega}\left(\mathbf{x}-\mathbf{z}_{\tilde{y}}\right)\left(\nu_{\tilde{\mathbf{y}}} \times \mathbf{E}^{i}\left(\mathbf{z}_{\tilde{y}}\right)\right) d \sigma_{\tilde{y}}=\int_{\Gamma_{0}} G_{\omega}\left(\mathbf{x}-\mathbf{z}_{\tilde{y}}\right) \int_{0}^{2 \pi} \nu_{\tilde{\mathbf{y}}} d \vartheta \times \mathbf{E}^{i}\left(\mathbf{z}_{\tilde{y}}\right) d r=0,
$$

where $(r, \vartheta)$ stand for the polar coordinates, and this together with $(3.32)$ readily implies (3.31).

Next, if $\Phi(\tilde{\mathbf{y}})=\Phi(\mathbf{y})=0$, then it can be seen from 3.21 and 3.32 that

$$
\|\tilde{\mathbf{a}}\|_{\mathrm{TH}^{-3 / 2}(\partial D)} \leq C,
$$

where $C$ is a positive constant depending only on $D$ and $\omega$. By using our earlier results in (3.27) and (3.28), one can first show that

$$
\begin{aligned}
\mathcal{S}_{D_{\delta}}^{\omega}[\mathbf{a}](\mathbf{x})= & \int_{\partial D_{\delta}} G_{\omega}\left(\mathbf{x}-\mathbf{z}_{y}\right) \mathbf{a}(\mathbf{y}) d \sigma_{y}-\int_{\partial D_{\delta}}\left(\nabla G_{\omega}\left(\mathbf{x}-\mathbf{z}_{y}\right) \cdot\left(\mathbf{y}-\mathbf{z}_{y}\right)\right) \mathbf{a}(\mathbf{y}) d \sigma_{y}+\mathcal{O}\left(\delta^{3}\right) \\
= & \delta \int_{S^{f}} G_{\omega}\left(\mathbf{x}-\mathbf{z}_{\tilde{y}}\right) \tilde{\mathbf{a}}(\tilde{\mathbf{y}}) d \sigma_{\tilde{y}}+\delta^{2}\left(\int_{S^{a} \cup S^{b}} G_{\omega}\left(\mathbf{x}-\mathbf{z}_{\tilde{y}}\right) \tilde{\mathbf{a}}(\tilde{\mathbf{y}}) d \sigma_{\tilde{y}}\right. \\
& \left.-\int_{S^{f}}\left(\nabla G_{\omega}\left(\mathbf{x}-\mathbf{z}_{\tilde{y}}\right) \cdot\left(\tilde{\mathbf{y}}-\mathbf{z}_{\tilde{y}}\right)\right) \tilde{\mathbf{a}}(\tilde{\mathbf{y}}) d \sigma_{\tilde{y}}\right)+\mathcal{O}\left(\delta^{3}\right)
\end{aligned}
$$

By using 3.32 we have for $\mathbf{y} \in S_{\delta}^{f}$ that

$$
\tilde{\mathbf{a}}(\tilde{\mathbf{y}})=2 \nu_{\tilde{\mathbf{y}}} \times \mathbf{E}^{i}\left(\mathbf{z}_{\tilde{y}}\right)+2 \delta \nu_{\tilde{\mathbf{y}}} \times\left(\nabla \mathbf{E}^{i}\left(\mathbf{z}_{\tilde{y}}\right)\left(\tilde{\mathbf{y}}-\mathbf{z}_{\tilde{y}}\right)\right)+\mathcal{O}\left(\delta^{2}\right) .
$$

Substituting (3.35) into (3.34) and using (3.33) one can easily obtain

$$
\begin{aligned}
\mathcal{S}_{D_{\delta}}^{\omega}[\mathbf{a}](\mathbf{x})=2 \delta^{2}( & \int_{S^{f}} G_{\omega}\left(\mathbf{x}-\mathbf{z}_{y}\right) \nu_{\tilde{\mathbf{y}}} \times\left(\nabla \mathbf{E}^{i}\left(\mathbf{z}_{\tilde{y}}\right)\left(\tilde{\mathbf{y}}-\mathbf{z}_{\tilde{y}}\right)\right) d \sigma_{y} \\
& +\int_{S^{a} \cup S^{b}} G_{\omega}\left(\mathbf{x}-\mathbf{z}_{\tilde{y}}\right) \nu_{\tilde{\mathbf{y}}} \times \mathbf{E}^{i}\left(\mathbf{z}_{\tilde{y}}\right) d \sigma_{\tilde{y}} \\
& \left.-\int_{S^{f}}\left(\nabla G_{\omega}\left(\mathbf{x}-\mathbf{z}_{\tilde{y}}\right) \cdot\left(\tilde{\mathbf{y}}-\mathbf{z}_{\tilde{y}}\right)\right) \nu_{\tilde{\mathbf{y}}} \times \mathbf{E}^{i}\left(\mathbf{z}_{\tilde{y}}\right)(\tilde{\mathbf{y}}) d \sigma_{\tilde{y}}\right)+\mathcal{O}\left(\delta^{3}\right),
\end{aligned}
$$

which then completes the proof by using (2.7).

We continue with the estimates of $R_{1}$ and $R_{2}$ in (3.27) for the proposed fullcloaking structure with arbitrary but regular $\varepsilon_{a}, \mu_{a}$ and $\sigma_{a}$ in $D_{\delta / 2}$. In what follows, we let $C$ denote a generic positive constant. It may change from one inequality to another inequality in our estimates. Moreover, it may depend on different parameters, but it does not depend on $\varepsilon_{a}, \mu_{a}, \sigma_{a}$ and $\mathbf{p}, \mathbf{d}, \hat{\mathbf{x}}$. 
We first note that, by using (3.32) and (3.21) in Lemma 3.4 there holds

$$
\|\tilde{\mathbf{a}}\|_{\mathrm{TH}^{-1 / 2}(\partial D)} \leq C\left(\|\tilde{\Phi}\|_{\mathrm{TH}^{-1 / 2}(\partial D)}+1\right) .
$$

Next, by taking expansion around $\mathbf{z} \in \Gamma_{0}$ and using (3.31) one can show that for $\delta \in \mathbb{R}_{+}$sufficiently small and $\|\mathbf{x}\|$ sufficiently large,

$$
\begin{aligned}
\left\|R_{1}\right\| & =\left\|\nabla \times \int_{\partial D_{\delta}} G_{\omega}\left(\mathbf{x}-\mathbf{z}_{y}\right) \mathbf{a}(\mathbf{y}) d \sigma_{y}\right\| \\
& \leq \delta\left\|\nabla \times \int_{S^{f}} G_{\omega}\left(\mathbf{x}-\mathbf{z}_{\tilde{y}}\right) \tilde{\mathbf{a}}(\tilde{\mathbf{y}}) d \sigma_{\tilde{y}}\right\|+C \delta^{2} \frac{1}{\|\mathbf{x}\|}\|\tilde{\mathbf{a}}\|_{\mathrm{TH}^{-1 / 2}(\partial D)} \\
& \leq C \delta\left\|\nabla \times \int_{S^{f}} G_{\omega}\left(\mathbf{x}-\mathbf{z}_{\tilde{y}}\right) \tilde{\Phi}(\tilde{\mathbf{y}}) d \sigma_{\tilde{y}}\right\|+C \delta^{2} \frac{1}{\|\mathbf{x}\|}\left(\|\tilde{\Phi}\|_{\mathrm{TH}^{-1 / 2}(\partial D)}+1\right) \\
& \leq C \delta \frac{1}{\|\mathbf{x}\|}\|\tilde{\Phi}\|_{\mathrm{TH}^{-1 / 2}\left(S^{f}\right)}+C \delta^{2} \frac{1}{\|\mathbf{x}\|}\left(\|\tilde{\Phi}\|_{\mathrm{TH}^{-1 / 2}(\partial D)}+1\right) .
\end{aligned}
$$

By using Taylor's expansions again and $(3.32)$, one can show the following estimation for $R_{2}$,

$$
\begin{aligned}
\left\|R_{2}\right\| & =\left\|\nabla \times \int_{\partial D_{\delta}} \nabla G_{\omega}\left(\mathbf{x}-\mathbf{z}_{y}\right) \cdot\left(\mathbf{y}-\mathbf{z}_{y}\right) \mathbf{a}(\mathbf{y}) d \sigma_{y}\right\| \\
& \leq C \delta^{2} \frac{1}{\|\mathbf{x}\|}\left(\|\tilde{\Phi}\|_{\mathrm{TH}^{-1 / 2}\left(S^{f}\right)}+1\right)+C \delta^{3} \frac{1}{\|\mathbf{x}\|}\left(\|\tilde{\Phi}\|_{\mathrm{TH}^{-1 / 2}(\partial D)}+1\right) .
\end{aligned}
$$

Hence, by applying the estimates in (3.28), 3.37) and (3.38) to (3.27), we have

$$
\left\|\mathbf{E}_{\delta}-\mathbf{E}^{i}\right\| \leq C \frac{\delta}{\|\mathbf{x}\|}\|\tilde{\Phi}\|_{\mathrm{TH}^{-1 / 2}\left(S^{f}\right)}+C \delta^{2} \frac{1}{\|\mathbf{x}\|}\left(\|\tilde{\Phi}\|_{\mathrm{TH}^{-1 / 2}(\partial D)}+1\right)
$$

for $\|\mathbf{x}\|$ sufficiently large. With (3.39) at hand, one readily has

Lemma 3.5. The scattering amplitude $\mathbf{A}_{\infty}^{\delta}(\hat{\mathbf{x}} ; \mathbf{p}, \mathbf{d})$ corresponding to the scattering configuration described in Theorem 3.1 satisfies

$$
\left\|\mathbf{A}_{\infty}^{\delta}(\hat{\mathbf{x}} ; \mathbf{p}, \mathbf{d})\right\| \leq C \delta\|\tilde{\Phi}\|_{\mathrm{TH}^{-1 / 2}\left(S^{f}\right)}+C \delta^{2}\left(\|\tilde{\Phi}\|_{\mathrm{TH}^{-1 / 2}(\partial D)}+1\right),
$$

where $C$ depends only on $D$ and $\omega$.

3.3. Proof of Theorem 3.1. By Lemma 3.5, it is straightforward to see that in order to derive the estimate of the scattering amplitude $\mathbf{A}_{\infty}^{\delta}$, it suffices for us to derive the corresponding estimate of $\|\tilde{\Phi}\|_{\mathrm{TH}^{-1 / 2}}$. To that end, we shall need the following lemma, whose proof can be found in 7 . 
Lemma 3.6. Let $B_{R}$ be a central ball of radius $R$ such that $D_{\delta} \Subset B_{R}$. Then the solutions $E_{\delta}, H_{\delta} \in H_{\text {loc }}\left(\operatorname{curl} ; \mathbb{R}^{3}\right)$ to 1.14$)$ verify

$$
\begin{aligned}
& \int_{D_{\delta} \backslash \bar{D}_{\delta / 2}} \sigma_{l} \mathbf{E}_{\delta} \cdot \overline{\mathbf{E}}_{\delta} d \mathbf{x}+\int_{D_{\delta / 2}} \sigma_{a} \mathbf{E}_{\delta} \cdot \overline{\mathbf{E}_{\delta}} d \mathbf{x} \\
= & \int_{\partial B_{R}}\left(\nu \times \overline{\mathbf{E}_{\delta}^{+}}\right) \cdot\left(\nu \times \nu \times \mathbf{H}_{\delta}^{+}\right) d \sigma_{x}+\left.\Re \int_{\partial D_{\delta}}\left(\nu \times \overline{\mathbf{E}^{i}}\right) \cdot\left(\nu \times \nu \times \mathbf{H}_{\delta}^{+}\right)\right|_{+} d \sigma_{x} \\
& +\left.\Re \int_{\partial D_{\delta}}\left(\nu \times \overline{\mathbf{E}_{\delta}^{+}}\right)\right|_{+} \cdot\left(\nu \times \nu \times \mathbf{H}^{i}\right) d \sigma_{x} .
\end{aligned}
$$

Remark 3.3. It is remarked that the last two terms in the RHS of (3.41) in [7] were

$$
\Re \int_{\partial B_{R}}\left(\nu \times \overline{\mathbf{E}^{i}}\right) \cdot\left(\nu \times \nu \times \mathbf{H}_{\delta}^{+}\right) d \sigma_{x}+\Re \int_{\partial B_{R}}\left(\nu \times \overline{\mathbf{E}_{\delta}^{+}}\right) \cdot\left(\nu \times \nu \times \mathbf{H}^{i}\right) d \sigma_{x} .
$$

Here, we modify the two terms for the convenience of the present study.

Define a boundary operator $\Lambda$ such that

$$
\Lambda\left(\nu \times\left.\mathbf{E}_{\delta}\right|_{\partial B_{R}}\right)=\nu \times\left.\mathbf{H}_{\delta}\right|_{\partial B_{R}}: \quad \mathrm{TH}_{\operatorname{div}}^{-1 / 2}\left(\partial B_{R}\right) \rightarrow \mathrm{TH}_{\operatorname{div}}^{-1 / 2}\left(\partial B_{R}\right) .
$$

It is well-known that $\Lambda$ is a bounded operator in $\mathrm{TH}_{\text {div }}^{-1 / 2}\left(\partial B_{R}\right)$ (cf. 10,31). By using Lemma 3.6, one can show that

Lemma 3.7. Let $\mathbf{E}_{\delta}$ and $\mathbf{H}_{\delta}$ be solutions to the system (1.14), where $D_{\delta}$ is the virtual domain described at the beginning of this section, then we have

$$
\int_{D_{\delta}^{f} \backslash \bar{D}_{\delta / 2}}\left\|\mathbf{E}_{\delta}\right\|^{2} d \mathbf{x} \leq C \delta^{1-t}\left(\left\|\nu \times \mathbf{E}_{\delta}^{+}\right\|_{\mathrm{TH}_{\mathrm{div}}^{-1 / 2}\left(\partial B_{R}\right)}^{2}+\delta\|\tilde{\mathbf{a}}\|_{\mathrm{TH}^{-1 / 2}(\partial D)}\right),
$$

and

$$
\int_{D_{\delta}^{c} \backslash \bar{D}_{\delta / 2}}\left\|\mathbf{E}_{\delta}\right\|^{2} d \mathbf{x} \leq C \delta^{2-t}\left(\left\|\nu \times \mathbf{E}_{\delta}^{+}\right\|_{\mathrm{TH}_{\mathrm{div}}^{-1 / 2}\left(\partial B_{R}\right)}^{2}+\delta\|\tilde{\mathbf{a}}\|_{\mathrm{TH}^{-1 / 2}(\partial D)}\right), c \in\{a, b\},
$$

where the constant $C$ depends only on $D, \omega$ and $R$.

Proof. First, by (3.41) and the fact that $\Lambda$ is bounded in $\mathrm{TH}_{\text {div }}^{-1 / 2}\left(\partial B_{R}\right)$, there holds

$$
\int_{D_{\delta} \backslash \bar{D}_{\delta / 2}} \sigma_{l} \mathbf{E}_{\delta} \cdot \overline{\mathbf{E}}_{\delta} d \mathbf{x} \leq C\left\|\nu \times \mathbf{E}_{\delta}^{+}\right\|_{\mathrm{TH}_{\mathrm{div}}^{-1 / 2}\left(\partial B_{R}\right)}^{2}+\mathbb{R}_{1}+\mathbb{R}_{2}
$$

where by using $(2.8)$ and $(3.22)$ one further has

$$
\begin{aligned}
\mathbb{R}_{1} & =\left.\left|\Re \int_{\partial D_{\delta}}\left(\nu \times \overline{\mathbf{E}^{i}}\right) \cdot\left(\nu \times \nu \times \mathbf{H}_{\delta}^{+}\right)\right|_{+} d \sigma_{x}|=| \Re \int_{\partial D_{\delta}} \overline{\mathbf{E}^{i}} \cdot\left(\nu \times \mathbf{H}_{\delta}^{+}\right)\right|_{+} d \sigma_{x} \mid \\
& \leq \frac{\delta}{\omega}\left|\int_{S^{a} \cup S^{b}} \overline{\mathbf{E}^{i}}\left(\mathbf{z}_{\tilde{\mathbf{x}}}\right) \cdot\left(\nu_{\tilde{\mathbf{x}}} \times \nabla_{\tilde{\mathbf{x}}} \times \nabla_{\tilde{\mathbf{x}}} \times \mathcal{A}_{S^{a} \cup S^{b}}[\tilde{\mathbf{a}}](\tilde{\mathbf{x}})\right) d \sigma_{\tilde{x}}\right|+C \delta^{2}\|\tilde{\mathbf{a}}\|_{\mathrm{TH}^{-1 / 2}(\partial D)},
\end{aligned}
$$


and by using (3.21) one further has

$$
\begin{aligned}
\mathbb{R}_{2} & =\left.\left|\Re \int_{\partial D_{\delta}}\left(\nu \times \overline{\mathbf{E}_{\delta}^{+}}\right)\right|_{+} \cdot\left(\nu \times \nu \times \mathbf{H}^{i}\right) d \sigma_{x}|=| \Re \int_{\partial D_{\delta}} \overline{\mathbf{E}_{\delta}^{+}}\right|_{+} \cdot\left(\nu \times \mathbf{H}^{i}\right) d \sigma_{x} \mid \\
& \leq C \delta^{2}\|\tilde{\mathbf{a}}\|_{\mathrm{TH}^{-1 / 2}(\partial D)} .
\end{aligned}
$$

Combining those with 3.9 , 3.11), together with the use of the definition of $\sigma_{l}$ in (3.7), we can complete the proof.

It is remarked that in the estimate (3.44), the dependence on the artificial $R$ of the generic constant $C$ can obviously be absorbed into the dependence on $D$.

In what follows, we shall estimate $\|\tilde{\Phi}\|_{\mathrm{TH}^{-1 / 2}\left(S^{f}\right)}$ and $\|\tilde{\Phi}\|_{\mathrm{TH}^{-1 / 2}\left(S^{a} \cup S^{b}\right)}$, separately. Clearly, it suffices to estimate $\|\tilde{\Phi}\|_{H^{-1 / 2}\left(S^{f}\right)^{3}}$ and $\|\tilde{\Phi}\|_{H^{-1 / 2}\left(S^{a} \cup S^{b}\right)^{3}}$, respectively. We recall that the $H^{-1 / 2}(\partial D)^{3}$-norm of the function $\tilde{\Phi}(\tilde{\mathbf{x}})$ is defined as follows

$$
\|\tilde{\Phi}\|_{H^{-1 / 2}(\partial D)^{3}}=\sup _{\|\varphi\|_{H^{1 / 2}(\partial D)^{3} \leq 1}}\left|\int_{\partial D} \tilde{\Phi}(\tilde{\mathbf{x}}) \cdot \bar{\varphi}(\tilde{\mathbf{x}}) d \sigma_{\tilde{x}}\right| .
$$

Moreover, the $H^{-1 / 2}\left(S^{c}\right)^{3}$-norm of $\tilde{\Phi}$ for $c \in\{f, a, b\}$ is given as

$$
\|\tilde{\Phi}\|_{H^{-1 / 2}\left(S^{c}\right)^{3}}:=\sup _{\|\varphi\|_{H_{0}^{1 / 2}\left(S^{c}\right)^{3}}}\left|\int_{S^{c}} \tilde{\Phi}(\tilde{\mathbf{x}}) \cdot \bar{\varphi}(\tilde{\mathbf{x}}) d \sigma_{\tilde{x}}\right|,
$$

where $H_{0}^{1 / 2}\left(S^{c}\right)^{3}$ denotes the set of $H^{1 / 2}\left(S^{c}\right)^{3}$-functions which have zero extensions to the whole boundary $\partial D$. We refer to [1,26, 35] for more relevant discussions on the Sobolev spaces. To proceed, we shall first establish the following important auxiliary Sobolev extension result, which is a localized version of Lemma 3.4 in [7].

Lemma 3.8. Suppose $D$ is a simply connected domain with a $C^{3}$-smooth boundary $\partial D$ and $S^{c} \Subset \partial D$ is an open surface lying on $\partial D$. Let $D^{\prime}$ be a simply connected domain with a $C^{3}$-smooth boundary $\partial D^{\prime}$ which satisfies $S^{c} \cap \partial D^{\prime}=\emptyset$ and $D^{\prime} \subset D$. Then for any $\psi \in H_{0}^{1 / 2}\left(S^{c}\right)^{3}$, there exists $\mathbf{W} \in H^{2}(D)^{3}$ such that

$$
\begin{array}{ll}
\nu \times \mathbf{W}=0 & \text { on } S^{c}, \\
\nu \times(\nu \times(\nabla \times \mathbf{W}))=\nu \times(\nu \times \psi) & \text { on } S^{c}, \\
\|\mathbf{W}\|_{H^{2}(D)^{3}} \leq C\|\psi\|_{H^{1 / 2}\left(S^{c}\right)^{3}}, & \\
\mathbf{W}=0 & \text { in } D^{\prime},
\end{array}
$$

where $C$ is a constant depending only on $D$.

Proof. By zero-extending $\psi$ to $\partial D$, we first have by Lemma 3.4 in [7] that there exists $\mathbf{W}_{0} \in H^{2}(D)^{3}$ satisifying

$$
\begin{array}{ll}
\nu \times \mathbf{W}_{0}=0 & \text { on } S^{c}, \\
\nu \times\left(\nu \times\left(\nabla \times \mathbf{W}_{0}\right)\right)=\nu \times(\nu \times \psi) & \text { on } S^{c}, \\
\left\|\mathbf{W}_{0}\right\|_{H^{2}(D)^{3}} \leq C\|\psi\|_{H^{1 / 2}\left(S^{c}\right)^{3}}, &
\end{array}
$$


where $C$ is a constant depending only on $D$. Let $\mathfrak{S}=\operatorname{supp}(\psi)$ denote the support of the function $\psi$. By definition there holds $\mathfrak{S} \Subset S^{c}$. Next, we use exactly the same strategy as that in Addendum of Theorem 14.1 in [35]. We cover $\mathfrak{S}$ with finitely many, say $\mathfrak{N}$, small enough regions $\left\{\mathfrak{S}_{j}\right\}_{j=1}^{\mathfrak{N}}$ such that $\left(\bigcup_{j=1}^{\mathfrak{N}} \mathfrak{S}_{j}\right) \cap \mathfrak{S} \subset S^{c}$. Then in each $\mathfrak{S}_{j} \cap D$ there exits an appropriately chosen $\mathbf{W}_{j} \in H^{2}\left(\left(\bigcup_{j=1}^{\mathfrak{N}} \mathfrak{S}_{j}\right) \cap D\right)^{3}$ (see formula (9) in Addendum in [35]) such that

$$
\begin{array}{ll}
\nu \times \mathbf{W}_{j}=0 & \text { on } \quad \partial\left(\mathfrak{S}_{j} \cap D\right) \cap S^{c}, \\
\nu \times\left(\nu \times\left(\nabla \times \mathbf{W}_{j}\right)\right)=\nu \times(\nu \times \psi) & \text { on } \quad \partial\left(\mathfrak{S}_{j} \cap D\right) \cap S^{c}, \\
\left\|\mathbf{W}_{j}\right\|_{H^{2}\left(\left(\bigcup_{j=1}^{\mathfrak{n}} \mathfrak{S}_{j}\right) \cap D\right)^{3}} \leq C\|\psi\|_{H^{1 / 2}\left(S^{c}\right)^{3}}, &
\end{array}
$$

where $C$ is a constant depending only on $D$. Finally, by setting $\mathbf{W}:=\sum_{j=1}^{\mathfrak{N}} \mathbf{W}_{j}$ in $\left(\bigcup_{j=1}^{\mathfrak{N}} \mathfrak{S}_{j}\right) \cap D$ and $\mathbf{W}=0$ in $D \backslash\left(\left(\bigcup_{j=1}^{\mathfrak{N}} \mathfrak{S}_{j}\right) \cap D\right)$, one can complete the proof.

We proceed with the proof of Theorem 3.1 .

Lemma 3.9. Let $\tilde{\Phi}$ be defined in (3.30), where $\left(\mathbf{E}_{\delta}, \mathbf{H}_{\delta}\right)$ are the solutions to (1.14) with the corresponding $\varepsilon_{\delta}, \mu_{\delta}$ and $\sigma_{\delta}$ given by (3.7). Then there hold

$$
\|\tilde{\Phi}\|_{\mathrm{TH}^{-1 / 2}\left(S^{f}\right)} \leq C \delta^{-1+\beta}\left\|\mathbf{E}_{\delta}\right\|_{L^{2}\left(D_{\delta}^{f} \backslash D_{\delta / 2}\right)^{3}},
$$

and

$$
\|\tilde{\Phi}\|_{\mathrm{TH}^{-1 / 2}\left(S^{c}\right)} \leq C \delta^{-3 / 2+\beta^{\prime}}\left\|\mathbf{E}_{\delta}\right\|_{L^{2}\left(D_{\delta}^{c} \backslash D_{\delta / 2}\right)^{3}}, \quad c \in\{a, b\},
$$

where $\beta=\min \{1,-1+r+s,-1+t+s\}$ and $\beta^{\prime}=\min \{1,-2+r+s,-2+t+s\}$.

Proof. It suffices to show that the same estimates in $(3.47)$ and $(3.48)$ hold for $\|\tilde{\Phi}(\tilde{\mathbf{x}})\|_{H^{-1 / 2}\left(S^{c}\right)^{3}}, c \in\{f, a, b\}$. For any test function $\psi \in H_{0}^{1 / 2}\left(S^{c}\right)^{3}, c \in\{f, a, b\}$, we introduce an auxiliary function $\mathbf{W} \in H^{2}(D)^{3}$ which satisfies the conditions in Lemma $3.8\left(D^{\prime}\right.$ is chosen to be $\left.D^{\prime}=D_{1 / 2} \cup\left(D \backslash D^{c}\right)\right)$. First, it follows from the properties of $\mathbf{W}$ that

$$
\begin{aligned}
& \int_{S^{f}} \tilde{\Phi}(\tilde{\mathbf{x}}) \cdot \psi(\tilde{\mathbf{x}}) d \sigma_{\tilde{x}}=-\int_{S^{f}}\left(\nu \times \tilde{\mathbf{E}}_{\delta}\right) \cdot(\nu \times(\nu \times \psi)) d \sigma_{\tilde{x}} \\
= & -\int_{S^{f}} \tilde{\mathbf{E}}_{\delta} \cdot(\nu \times(\nabla \times \mathbf{W})) d \sigma_{\tilde{x}}-\int_{\partial D^{f} \backslash S^{f}} \tilde{\mathbf{E}}_{\delta} \cdot(\nu \times(\nabla \times \mathbf{W})) d \sigma_{\tilde{x}} \\
= & -\int_{\partial D^{f}} \tilde{\mathbf{E}}_{\delta} \cdot(\nu \times(\nabla \times \mathbf{W})) d \sigma_{\tilde{x}} .
\end{aligned}
$$

From its construction, it is readily seen that $\nu \times\left.\mathbf{W}\right|_{\partial D^{c}}=0$. Define

$$
\widehat{\operatorname{Curl} W}:=\left(\tilde{\mathbf{B}}^{T}\right)^{-1}(\nabla \times \mathbf{W}) .
$$


By using (3.16), 3.19), 3.12 and integration by parts, one also has

$$
\begin{aligned}
& \int_{S^{f}} \tilde{\Phi}(\tilde{\mathbf{x}}) \cdot \psi(\tilde{\mathbf{x}}) d \sigma_{\tilde{x}}=-\delta^{-1} \int_{\partial D^{f}} \hat{\mathbf{E}}_{\delta} \cdot(\nu \times \widehat{\operatorname{Curl} W}) d \sigma_{\tilde{x}} \\
= & -\delta^{-1}\left(\int_{\partial D^{f}} \hat{\mathbf{E}}_{\delta} \cdot(\nu \times \widehat{\operatorname{Curl} W}) d \sigma_{\tilde{x}}-\int_{\partial D^{f}} \mathbf{W} \cdot(\nu \times \widehat{\operatorname{Curl} W}) d \sigma_{\tilde{x}}\right) \\
= & \left.\delta^{-1}\left(\int_{D^{f}} \mathbf{W} \cdot(\nabla \times \widehat{\operatorname{Curl} W}) d \tilde{\mathbf{x}}-\int_{D^{f}} \hat{\mathbf{E}}_{\delta} \cdot(\nabla \times \widehat{\operatorname{Curl} W})\right) d \tilde{\mathbf{x}}\right) \\
= & \delta^{-1} \int_{D^{f} \backslash \bar{D}_{1 / 2}} \mathbf{W} \cdot(\nabla \times \widehat{\operatorname{Curl} W}) d \tilde{\mathbf{x}}-\delta \int_{D^{f} \backslash \bar{D}_{1 / 2}} \tilde{\mathbf{E}}_{\delta} \cdot(\nabla \times(\nabla \times \mathbf{W})) d \tilde{\mathbf{x}} .
\end{aligned}
$$

For $\mathbf{x} \in D_{\delta} \backslash D_{\delta / 2}$ there holds

$$
\nabla_{\mathbf{x}} \times \mathbf{E}_{\delta}=i \omega \mu_{l} \mathbf{H}_{\delta}, \quad \nabla_{\mathbf{x}} \times \mathbf{H}_{\delta}=-i \omega\left(\varepsilon_{l}+i \frac{\sigma_{l}}{\omega}\right) \mathbf{E}_{\delta}
$$

By using change of variables and $(3.16)$ in Lemma 3.2 , one can derive that

$$
|\tilde{\mathbf{B}}| \tilde{\mathbf{B}}^{-1} \nabla_{\tilde{\mathbf{x}}} \times \hat{\mathbf{E}}_{\delta}=i \omega \tilde{\mu}_{l} \tilde{\mathbf{H}}_{\delta}, \quad|\tilde{\mathbf{B}}| \tilde{\mathbf{B}}^{-1} \nabla_{\tilde{\mathbf{x}}} \times \hat{\mathbf{H}}_{\delta}=-i \omega\left(\tilde{\varepsilon}_{l}+i \frac{\tilde{\sigma}_{l}}{\omega}\right) \tilde{\mathbf{E}}_{\delta},
$$

which holds in $D \backslash \bar{D}_{1 / 2}$. By combining (3.7), (3.15) and $(3.49)$, one can further show that in $D \backslash \bar{D}_{1 / 2}$,

$$
\nabla \times \widehat{\operatorname{Curl} W}=\omega^{2}\left(\delta^{r+s}+i \delta^{t+s} \frac{1}{\omega}\right) \tilde{\mathbf{E}}_{\delta} .
$$

Thus by using (3.50) one has

$$
\begin{aligned}
& \int_{S^{f}} \tilde{\Phi}(\tilde{\mathbf{x}}) \cdot \psi(\tilde{\mathbf{x}}) d \sigma_{\tilde{x}} \\
= & \omega^{2}\left(\delta^{-1+r+s}+i \delta^{-1+t+s} \frac{1}{\omega}\right) \int_{D^{f} \backslash \bar{D}_{1 / 2}} \mathbf{W} \cdot \tilde{\mathbf{E}}_{\delta} d \tilde{\mathbf{x}}-\delta \int_{D^{f} \backslash \bar{D}_{1 / 2}} \tilde{\mathbf{E}}_{\delta} \cdot(\nabla \times(\nabla \times \mathbf{W})) d \tilde{\mathbf{x}},
\end{aligned}
$$

which in combination with the fact that

$$
\left\|\tilde{\mathbf{E}}_{\delta}\right\|_{L^{2}\left(D^{f} \backslash \bar{D}_{1 / 2}\right)^{3}}=\delta^{-1}\left\|\mathbf{E}_{\delta}\right\|_{L^{2}\left(D_{\delta}^{f} \backslash \bar{D}_{\delta / 2}\right)^{3}},
$$

immediately yields that

$$
\begin{aligned}
\left|\int_{S^{c}} \tilde{\Phi}(\tilde{\mathbf{x}}) \cdot \psi(\tilde{\mathbf{x}}) d \sigma_{\tilde{x}}\right| & \leq C \delta^{\beta}\left\|\tilde{\mathbf{E}}_{\delta}\right\|_{L^{2}\left(D^{f} \backslash D_{1 / 2}\right)^{3}}\|\mathbf{W}\|_{H^{2}\left(D^{f} \backslash D_{1 / 2}\right)^{3}} \\
& \leq C \delta^{-1+\beta}\left\|\mathbf{E}_{\delta}\right\|_{L^{2}\left(D_{\delta}^{f} \backslash D_{\delta / 2}\right)^{3}}\|\mathbf{W}\|_{H^{2}\left(D^{f} \backslash D_{1 / 2}\right)^{3}}
\end{aligned}
$$


where $\beta=\min \{1,-1+r+s,-1+t+s\}$. By using exactly the same strategy as above with $S^{f}$ and 3.20 , one can show that for $c \in\{a, b\}$

$$
\begin{aligned}
& \int_{S^{c}} \tilde{\Phi}(\tilde{\mathbf{x}}) \cdot \psi(\tilde{\mathbf{x}}) d \sigma_{\tilde{x}} \\
= & \delta^{-2} \int_{D^{c} \backslash \bar{D}_{1 / 2}} \mathbf{W} \cdot(\nabla \times \widehat{\operatorname{Curl} W}) d \tilde{\mathbf{x}}-\delta \int_{D^{c} \backslash \bar{D}_{1 / 2}} \tilde{\mathbf{E}}_{\delta} \cdot(\nabla \times(\nabla \times \mathbf{W})) d \tilde{\mathbf{x}},
\end{aligned}
$$

which together with the fact

$$
\left\|\tilde{\mathbf{E}}_{\delta}\right\|_{L^{2}\left(D^{c} \backslash \bar{D}_{1 / 2}\right)^{3}}=\delta^{-3 / 2}\left\|\mathbf{E}_{\delta}\right\|_{L^{2}\left(D_{\delta}^{c} \backslash \bar{D}_{\delta / 2}\right)^{3}}, \quad c \in\{a, b\},
$$

readily yields that

$$
\left|\int_{S^{c}} \tilde{\Phi}(\tilde{\mathbf{x}}) \cdot \psi(\tilde{\mathbf{x}}) d \sigma_{\tilde{x}}\right| \leq C \delta^{-3 / 2+\beta^{\prime}}\left\|\mathbf{E}_{\delta}\right\|_{L^{2}\left(D_{\delta}^{c} \backslash \bar{D}_{\delta / 2}\right)^{3}}\|\mathbf{W}\|_{H^{2}\left(D^{c} \backslash \bar{D}_{1 / 2}\right)^{3}}
$$

where $\beta^{\prime}=\min \{1,-2+r+s,-2+t+s\}$. The proof can be completed by noting the definitions 3.45, 3.46) and the fact that

$$
\|\mathbf{W}\|_{H^{2}\left(D^{c} \backslash \bar{D}_{1 / 2}\right)^{3}} \leq C\|\psi\|_{H^{1 / 2}\left(\partial D^{c}\right)^{3}}, \quad c \in\{f, a, b\} .
$$

Proof of Theorem 3.1. It is straightforward to see from 3.40 that we only need to derive the estimates of $\|\tilde{\Phi}\|_{\mathrm{TH}^{-1 / 2}\left(S^{f}\right)}$ and $\|\tilde{\Phi}\|_{\mathrm{TH}^{-1 / 2}(\partial D)}$. To that end, we have by using (2.7), 3.13), 3.35) and (3.36) that

$$
\begin{aligned}
\left\|\nu \times \mathbf{E}_{\delta}^{+}\right\|_{\mathrm{TH}_{\mathrm{div}}^{-1 / 2}\left(\partial B_{R}\right)} & \leq C\left\|\nu \times \nabla \times \mathcal{A}_{D_{\delta}}[\mathbf{a}]\right\|_{\mathrm{TH}_{\mathrm{div}}^{-1 / 2}\left(\partial B_{R}\right)} \\
& \leq C \delta\|\tilde{\mathbf{a}}\|_{\mathrm{TH}^{-1 / 2}\left(S^{f}\right)}+C \delta^{2}\|\tilde{\mathbf{a}}\|_{\mathrm{TH}^{-1 / 2}(\partial D)} \\
& \leq C \delta\|\tilde{\Phi}\|_{\mathrm{TH}^{-1 / 2}\left(S^{f}\right)}+C \delta^{2}\left(\|\tilde{\Phi}\|_{\mathrm{TH}^{-1 / 2}(\partial D)}+1\right) .
\end{aligned}
$$

Next, by combining (3.51), (3.36), (3.43), (3.44), (3.47) and (3.48), together with the use of the facts that both $\Lambda$ and $\Lambda^{-}$are bounded, and $\beta^{\prime} \leq \beta$ (see Lemma 3.9), one can further deduce that

$$
\begin{aligned}
\|\tilde{\Phi}\|_{\mathrm{TH}^{-1 / 2}(\partial D) \leq} \leq & C \delta^{-1+\beta}\left\|\mathbf{E}_{\delta}\right\|_{L^{2}\left(D_{\delta}^{f} \backslash D_{\delta / 2}\right)^{3}}+C \delta^{-3 / 2+\beta^{\prime}}\left\|\mathbf{E}_{\delta}\right\|_{L^{2}\left(D_{\delta}^{a \cup b} \backslash D_{\delta / 2}\right)^{3}} \\
\leq & C \delta^{-1+\beta+1 / 2-t / 2}\left(\left\|\nu \times \mathbf{E}_{\delta}^{+}\right\|_{\mathrm{TH}_{\mathrm{div}}^{-1 / 2}\left(\partial B_{R}\right)}^{2}+\mathcal{O}\left(\delta\|\tilde{\mathbf{a}}\|_{\mathrm{TH}^{-1 / 2}(\partial D)}\right)\right)^{1 / 2} \\
& +C \delta^{-3 / 2+\beta^{\prime}+1-t / 2}\left(\left\|\nu \times \mathbf{E}_{\delta}^{+}\right\|_{\mathrm{TH}_{\mathrm{div}}^{-1 / 2}\left(\partial B_{R}\right)}^{2}+\mathcal{O}\left(\delta\|\tilde{\mathbf{a}}\|_{\mathrm{TH}^{-1 / 2}(\partial D)}\right)\right)^{1 / 2} \\
\leq & C \delta^{\beta^{\prime}-t / 2-1 / 2}\left(\delta\|\tilde{\Phi}\|_{\mathrm{TH}^{-1 / 2}\left(S^{f}\right)}+\delta^{2}\|\tilde{\Phi}\|_{\mathrm{TH}^{-1 / 2}(\partial D)}+\delta^{2}\right) \\
& +C \delta^{\beta^{\prime}-t / 2}\left(\|\tilde{\Phi}\|_{\mathrm{TH}^{-1 / 2}(\partial D)}^{1 / 2}+1\right),
\end{aligned}
$$

which in turn yields (noting that $\beta^{\prime}-t / 2 \geq 0$ )

$$
\|\tilde{\Phi}\|_{\mathrm{TH}^{-1 / 2}(\partial D)} \leq C \delta^{\beta^{\prime}-t / 2}\left(\|\tilde{\Phi}\|_{\mathrm{TH}^{-1 / 2}(\partial D)}^{1 / 2}+1\right) .
$$


Thus one has

$$
\|\tilde{\Phi}\|_{\mathrm{TH}^{-1 / 2}(\partial D)} \leq C \delta^{\beta^{\prime}-t / 2} .
$$

It is remarked that in (3.52), the generic constant obviously depends on $R$, but as was remarked earlier that such a dependence can be absorbed into the dependence on $D$. Next, by using (3.47) and (3.52), there holds

$$
\begin{aligned}
\|\tilde{\Phi}\|_{\mathrm{TH}^{-1 / 2}\left(S^{f}\right)} & \leq C \delta^{-1+\beta}\left\|\mathbf{E}_{\delta}\right\|_{L^{2}\left(D_{\delta}^{f} \backslash D_{\delta / 2}\right)^{3}} \\
& \leq C \delta^{-1+\beta+1 / 2-t / 2}\left(\left\|\nu \times \mathbf{E}_{\delta}^{+}\right\|_{\mathrm{TH}_{\mathrm{div}}^{-1 / 2}\left(\partial B_{R}\right)}^{2}+\mathcal{O}\left(\delta\|\tilde{\mathbf{a}}\|_{\mathrm{TH}^{-1 / 2}(\partial D)}\right)\right)^{1 / 2} \\
& \leq C \delta^{-1 / 2+\beta-t / 2}\left(\delta\|\tilde{\Phi}\|_{\mathrm{TH}^{-1 / 2}\left(S^{f}\right)}+\delta^{2}\right)+C \delta^{\beta-t / 2}
\end{aligned}
$$

and thus

$$
\|\tilde{\Phi}\|_{\mathrm{TH}^{-1 / 2}\left(S^{f}\right)} \leq C \delta^{\beta-t / 2} .
$$

By plugging $(3.52)$ and $(3.53)$ into $(3.40)$, one finally has $(3.8)$.

The proof is complete.

\section{Regularized PARTial-ClOAKING OF EM WAVES}

In this section, we consider the regularized partial-cloaking of EM waves by taking the generating set $\Gamma_{0}$ to be a flat subset on a plane in $\mathbb{R}^{3}$. In order to ease our exposition, we stick our subsequent study to a specific example considered in 9.25 for the partial-cloaking of acoustic waves, where $\Gamma_{0}$ is taken to be a square on $\mathbb{P}_{2}:=\left\{\mathbf{x}=\left(x_{1}, x_{2}, x_{3}\right) \in \mathbb{R}^{3}: x_{3}=0\right\}$. The virtual domain is constructed as follows; see Fig. 2 for a schematic illustration. Let $\mathbf{n} \in \mathbb{S}^{2}$ be the unit normal vector to $\Gamma_{0}$. Let

$$
D_{q}^{0}:=\Gamma_{0}(\mathbf{x}) \times[\mathbf{x}-\tau \cdot \mathbf{n}, \mathbf{x}+\tau \cdot \mathbf{n}], \quad \mathbf{x} \in \bar{\Gamma}_{0}, \quad 0 \leq \tau \leq q,
$$

where we identify $\Gamma_{0}$ with its parametric representation $\Gamma_{0}(\mathbf{x})$. We denote by $D_{q}^{1}$ the union of the four side half-cylinders and $D_{q}^{2}$ the union of the four corner quarter-balls in Fig. 2, Let

$$
D_{q}:=D_{q}^{0} \cup D_{q}^{1} \cup D_{q}^{2}
$$

Set $S_{q}^{1}$ and $S_{q}^{2}$ to denote

$$
S_{q}^{1}:=\partial D_{q} \cap \partial D_{q}^{1}, \quad S_{q}^{2}:=\partial D_{q} \cap \partial D_{q}^{2},
$$

and

$$
D_{q}^{f}:=D_{q}^{1} \cup D_{q}^{2} .
$$

The upper and lower-surfaces of $D_{q}$ are respectively denoted by

$$
\Gamma_{q}^{1}:=\left\{\mathbf{x}+q \cdot \mathbf{n} ; \mathbf{x} \in \Gamma_{0}\right\} \quad \text { and } \quad \Gamma_{q}^{2}:=\left\{\mathbf{x}-q \cdot \mathbf{n} ; \mathbf{x} \in \Gamma_{0}\right\} .
$$


Define $S_{q}^{0}:=\Gamma_{q}^{1} \cup \Gamma_{q}^{2}$. We then have $\partial D_{q}=S_{q}^{0} \cup S_{q}^{1} \cup S_{q}^{2}$. Similar to our notations in Section 3 , we let $\delta \in \mathbb{R}_{+}$denote the asymptotically small regularization parameter and $D_{\delta}$ be the virtual domain. Clearly, we have

$$
\partial D_{\delta}=S_{\delta}^{0} \cup S_{\delta}^{1} \cup S_{\delta}^{2} .
$$

In what follows, if $q \equiv 1$, we drop the dependence on $q$ of $D_{q}, S_{q}^{0}, S_{q}^{1}$ and $S_{q}^{2}$, and simply write them as $D, S^{0}, S^{1}$, and $S^{2}$. In concluding the description of the virtual domain for the partial-cloaking construction, we would like to emphasize that our subsequent study can be easily extended to a much more general case where the generating set $\Gamma_{0}$ can be a bounded subset on a plane with a convex and piecewise smooth boundary (in the topology induced from the plane).
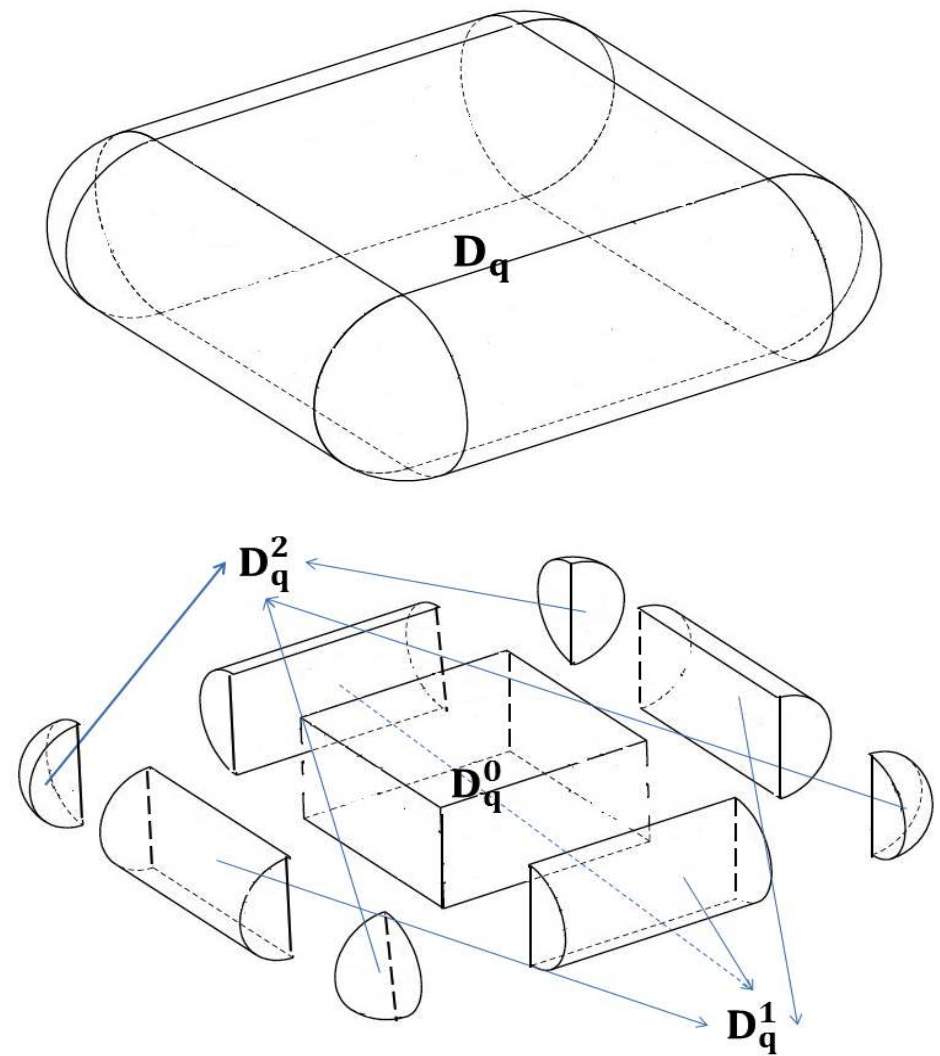

Figure 2. Schematic illustration of the domain $D_{q}$ for the regularized partial-cloak.

We introduce a blowup transformation $A$ which maps $D_{\delta}$ to $D$ exactly as that in [25]. We stress that in $D_{\delta}^{0}$ the blow-up transformation takes the following form

$$
A(\mathbf{y})=\tilde{\mathbf{y}}:=\left(\frac{\mathbf{e}_{3} \mathbf{e}_{3}^{T}}{\delta}+\mathbf{e}_{1} \mathbf{e}_{1}^{T}+\mathbf{e}_{2} \mathbf{e}_{2}^{T}\right) \mathbf{y}, \quad \mathbf{y} \in D_{\delta}^{0} .
$$


where $\mathbf{y} \in D_{\delta}$ and $\tilde{\mathbf{y}} \in D$, and the three Euclidean unit vectors are as follows

$$
\mathbf{e}_{1}=(1,0,0)^{T}, \quad \mathbf{e}_{2}=(0,1,0)^{T}, \quad \mathbf{e}_{3}=(0,0,1)^{T} .
$$

We are now in the position of introducing the lossy layer for our partial-cloaking device

$$
\begin{aligned}
& \varepsilon_{\delta}(\mathbf{x})=\varepsilon_{l}(\mathbf{x}):=\delta^{r}|\mathbf{B}| \mathbf{B}^{-1}, \quad \mu_{\delta}(\mathbf{x})=\mu_{l}(\mathbf{x}):=\delta^{s}|\mathbf{B}| \mathbf{B}^{-1}, \\
& \sigma_{\delta}(\mathbf{x})=\sigma_{l}(\mathbf{x}):=\delta^{t}|\mathbf{B}| \mathbf{B}^{-1}, \quad \text { for } \quad \mathbf{x} \in D_{\delta} \backslash \bar{D}_{\delta / 2},
\end{aligned}
$$

where $\mathbf{B}(\mathbf{x}):=\nabla_{\mathbf{x}} A(\mathbf{x})$ is the Jacobian matrix of the blowup transformation $A$. It is obvious that

$$
\mathbf{B}=\frac{\mathbf{e}_{3} \mathbf{e}_{3}^{T}}{\delta}+\mathbf{e}_{1} \mathbf{e}_{1}^{T}+\mathbf{e}_{2} \mathbf{e}_{2}^{T} \quad \text { in } \quad D_{\delta}^{0},
$$

is a constant matrix and

$$
\mathbf{B n}=\frac{\mathbf{n}}{\delta}, \quad \mathbf{B e}_{1}=\mathbf{e}_{1}, \quad \mathbf{B} \mathbf{e}_{2}=\mathbf{e}_{2} \quad \text { in } \quad D_{\delta}^{0} .
$$

Thus one has

$$
|\mathbf{B}|=1 / \delta, \quad \text { in } D_{\delta}^{0} .
$$

Furthermore, for $\mathbf{x} \in D_{\delta}$, one can show by direct calculations that 3.10 and (3.11) are also valid for the $\mathbf{B}$ defined above.

Lemma 4.1. Let $D^{j}, j=0,1,2$ be defined at the beginning of this section. Let $\mathbf{V}$, $\mathbf{W}$ and $\hat{\mathbf{V}}, \hat{\mathbf{W}}$ be defined similarly as those in Lemma 3.2. Then one has

$$
\int_{\partial D^{j}}\left(\nu_{\tilde{\mathbf{x}}} \times \tilde{\mathbf{V}}(\tilde{\mathbf{x}})\right) \cdot \mathbf{W}(\tilde{\mathbf{x}}) d \sigma_{\tilde{x}}=\delta^{-j} \int_{\partial D^{j}}\left(\nu_{\tilde{\mathbf{x}}} \times \hat{\mathbf{V}}(\tilde{\mathbf{x}})\right) \cdot \hat{\mathbf{W}}(\tilde{\mathbf{x}}) d \sigma_{\tilde{x}}, \quad j=0,1,2 .
$$

We are now in a position to present the main theorem of this section in quantifying our partial-cloaking construction.

Theorem 4.1. Let $D_{\delta}$ be defined in (4.2) with its boundary given by (4.3). Let $\left(\mathbf{E}_{\delta}, \mathbf{H}_{\delta}\right)$ be the pair of solutions to $(1.14)$ with $\left\{\Omega ; \varepsilon_{\delta}, \mu_{\delta}, \sigma_{\delta}\right\} \subset\left\{\mathbb{R}^{3} ; \varepsilon_{\delta}, \mu_{\delta}, \sigma_{\delta}\right\}$ defined in (1.3) and $\left\{D_{\delta} \backslash \bar{D}_{\delta / 2} ; \varepsilon_{\delta}, \mu_{\delta}, \sigma_{\delta}\right\}$ given in (4.4). Let $\mathbf{A}_{\infty}^{\delta}(\hat{\mathbf{x}} ; \mathbf{p}, \mathbf{d})$ be the scattering amplitude of $\mathbf{E}_{\delta}$. Define

$$
\beta_{j}=\min \{1,-j+r+s,-j+t+s\}, j=0,1,2,
$$

and for $\epsilon \in \mathbb{R}_{+}$with $\epsilon \ll 1$,

$$
\Sigma_{p}:=\left\{\mathbf{p} \in \mathbb{S}^{2} ;\|\mathbf{p} \times \mathbf{n}\| \leq \epsilon\right\}, \quad \Sigma_{d}:=\left\{\mathbf{d} \in \mathbb{S}^{2} ;|\mathbf{d} \cdot \mathbf{n}| \leq \epsilon\right\} .
$$

If $r, s$ and $t$ are chosen such that $\beta_{2}-t / 2>0$, then there exists $\delta_{0} \in \mathbb{R}_{+}$such that when $\delta<\delta_{0}$,

$$
\left\|\mathbf{A}_{\infty}^{\delta}(\hat{\mathbf{x}} ; \mathbf{p}, \mathbf{d})\right\| \leq C\left(\epsilon+\delta^{2\left(\beta_{2}-t / 2\right)}+\delta\right), \quad \mathbf{p} \in \Sigma_{p}, \mathbf{d} \in \Sigma_{d}, \hat{\mathbf{x}} \in \mathbb{S}^{2},
$$

where $C$ is a positive constant depending on $\omega$ and $D$, but independent of $\varepsilon_{a}, \mu_{a}$, $\sigma_{a}$ and $\hat{\mathbf{x}}, \mathbf{p}, \mathbf{d}$. 
Remark 4.1. Similar to Remark 3.1, one readily has from Theorems 1.1 and 4.1 that the push-forwarded structure in (1.2) produces an approximate partial-cloaking device which is capable of nearly cloaking an arbitrary EM content. The highest accuracy of such a construction can be achieved, say for example, by taking $r=$ $t=0$ and $s=5 / 2$.

Remark 4.2. Since $\mathbf{p} \cdot \mathbf{d}=0$ (cf. (1.7)), one has

$$
(\mathbf{d} \cdot \mathbf{n}) \mathbf{p}=\mathbf{d} \times(\mathbf{p} \times \mathbf{n}),
$$

from which one can infer that the definition of $\Sigma_{d}$ in 4.7 is redundant. However, we specified it for clarity and definiteness.

4.1. Auxiliary lemmas and asymptotic expansions. We shall follow a similar strategy of the proof for Theorem 3.1 in proving Theorem 4.1. In what follows, we adopt similar notations as those in Section 3 . If we let $\mathbf{z}$ denote the space variable on $\Gamma_{0}$, then for any $\mathbf{y} \in \partial D_{q}$, we define $\mathbf{z}_{y}$ to be the projection of $\mathbf{y}$ onto $\Gamma_{0}$. We also define $\tilde{\mathbf{a}}(\tilde{\mathbf{y}}):=\mathbf{a}(\mathbf{y})$ and $\tilde{\mathbf{y}}:=A(\mathbf{y}) \in \bar{D}$ for $\mathbf{y} \in \bar{D}_{\delta}$.

The following lemma is a counterpart to Lemma 3.4 in Section 3.

Lemma 4.2. Let $D_{\delta}$ be described in (4.2) and (4.3). Define

$$
\mathcal{M}_{S^{0}}^{\omega}[\tilde{\mathbf{a}}](\tilde{\mathbf{x}}):=\text { p.v. } \quad \nu_{\tilde{\mathbf{x}}} \times \nabla_{\mathbf{z}_{\tilde{x}}} \times \int_{S^{0}} G_{\omega}\left(\mathbf{z}_{\tilde{x}}-\mathbf{z}_{\tilde{y}}\right) \tilde{\mathbf{a}}(\tilde{\mathbf{y}}) d \sigma_{\tilde{y}},
$$

and

$$
\mathcal{M}_{S^{2}}[\tilde{\mathbf{a}}](\tilde{\mathbf{x}}):=\text { p.v. } \quad \nu_{\tilde{\mathbf{x}}} \times \nabla_{\tilde{\mathbf{x}}} \times \int_{S^{2}} G_{\omega}(\tilde{\mathbf{x}}-\tilde{\mathbf{y}}) \tilde{\mathbf{a}}(\tilde{\mathbf{y}}) d \sigma_{\tilde{y}}
$$

Define

$$
\mathcal{L}_{D_{\delta}}^{\omega}[\mathbf{a}](\mathbf{x}):=\nu_{\mathbf{x}} \times \nabla_{\mathbf{x}} \times \nabla_{\mathbf{x}} \times \mathcal{A}_{D_{\delta}}^{\omega}[\mathbf{a}](\mathbf{x})
$$

and

$$
\mathcal{L}_{S^{0}}^{\omega}[\tilde{\mathbf{a}}](\mathbf{x}):=\nu_{\tilde{\mathbf{x}}} \times \nabla_{\mathbf{z}_{\tilde{x}}} \times \nabla_{\mathbf{z}_{\tilde{x}}} \times \int_{S^{0}} G_{\omega}\left(\mathbf{z}_{\tilde{x}}-\mathbf{z}_{\tilde{y}}\right) \tilde{\mathbf{a}}(\tilde{\mathbf{y}}) d \sigma_{\tilde{y}} .
$$

Then one has

$$
\mathcal{M}_{D_{\delta}}^{\omega}[\mathbf{a}](\mathbf{x})= \begin{cases}\mathcal{M}_{S^{0}}^{\omega}[\tilde{\mathbf{a}}](\tilde{\mathbf{x}})+\mathcal{O}\left(\delta\|\tilde{\mathbf{a}}\|_{\mathrm{TH}^{-1 / 2}(\partial D)}\right), & \mathbf{x} \in S_{\delta}^{0} \cup S_{\delta}^{1}, \\ \mathcal{M}_{S^{0}}^{\omega}[\tilde{\mathbf{a}}](\tilde{\mathbf{x}})+\mathcal{M}_{S^{2}}[\tilde{\mathbf{a}}](\tilde{\mathbf{x}})+\mathcal{O}\left(\delta\|\tilde{\mathbf{a}}\|_{\mathrm{TH}^{-1 / 2}(\partial D)}\right), & \mathbf{x} \in S_{\delta}^{2},\end{cases}
$$

and

$$
\mathcal{L}_{D_{\delta}}^{\omega}[\mathbf{a}](\mathbf{x})= \begin{cases}\mathcal{L}_{S^{0}}^{\omega}[\tilde{\mathbf{a}}](\tilde{\mathbf{x}})+\mathcal{O}\left(\delta\|\tilde{\mathbf{a}}\|_{\mathrm{TH}^{-1 / 2}(\partial D)}\right), & \mathbf{x} \in S_{\delta}^{0} \cup S_{\delta}^{1} \\ \frac{1}{\delta} \nu_{\tilde{\mathbf{x}}} \times \nabla_{\tilde{\mathbf{x}}} \times \nabla_{\tilde{\mathbf{x}}} \times \mathcal{A}_{S^{2}}[\tilde{\mathbf{a}}](\tilde{\mathbf{x}})+\mathcal{O}\left(\|\tilde{\mathbf{a}}\|_{\mathrm{TH}^{-1 / 2}(\partial D)}\right), & \mathbf{x} \in S_{\delta}^{2}\end{cases}
$$

Proof. By following a similar argument to that in the proof of Lemma 3.4, one can compute for $\mathbf{x} \in S_{\delta}^{0} \cup S_{\delta}^{1}$ that

$$
\|\mathbf{x}-\mathbf{y}\|=\left\|\mathbf{x}-\mathbf{z}_{x}-\left(\mathbf{y}-\mathbf{z}_{y}\right)+\mathbf{z}_{x}-\mathbf{z}_{y}\right\|=\left\|\mathbf{z}_{\tilde{x}}-\mathbf{z}_{\tilde{y}}\right\|+\mathcal{O}(\delta),
$$


and

$$
\left\langle\mathbf{x}-\mathbf{y}, \nu_{\mathbf{x}}\right\rangle=\left\langle\mathbf{z}_{x}-\mathbf{z}_{y}, \nu_{\tilde{\mathbf{x}}}\right\rangle+\mathcal{O}(\delta), \quad e^{i \omega|\mathbf{x}-\mathbf{y}|}=e^{i \omega\left|\mathbf{z}_{\tilde{x}}-\mathbf{z}_{\tilde{y}}\right|}+\mathcal{O}(\delta) .
$$

One also notes that for $\mathbf{x} \in S_{\delta}^{2}$

$$
\|\mathbf{x}-\mathbf{y}\|=\delta\|\tilde{\mathbf{x}}-\tilde{\mathbf{y}}\|, \quad e^{i \omega\|\mathbf{x}-\mathbf{y}\|}=1+\mathcal{O}(\delta) .
$$

By using the above facts, the proof can then be completed by using the similar expansion method as that in the proof of Lemma 3.4.

Next, by straightforward calculations, one can show that for $D_{\delta}$ described in (4.2) and (4.3), there holds the following far field expansion for $\mathbf{x} \in \mathbb{R}^{3} \backslash \bar{D}_{\delta}$,

$$
\begin{aligned}
\mathcal{S}_{D_{\delta}}^{\omega}[\mathbf{a}](\mathbf{x})= & \int_{\partial D_{\delta}} G_{\omega}(\mathbf{x}-\mathbf{y}) \mathbf{a}(\mathbf{y}) d \sigma_{y}=\int_{S^{0}} G_{\omega}\left(\mathbf{x}-\mathbf{z}_{\tilde{y}}\right) \tilde{\mathbf{a}}(\tilde{\mathbf{y}}) d \sigma_{\tilde{y}} \\
& +\delta \int_{S^{1}} G_{\omega}\left(\mathbf{x}-\mathbf{z}_{\tilde{y}}\right) \tilde{\mathbf{a}}(\tilde{\mathbf{y}}) d \sigma_{\tilde{y}}+\delta \int_{S^{0}}\left(\tilde{\mathbf{y}}-\mathbf{z}_{\tilde{y}}\right) \cdot \nabla G_{\omega}\left(\mathbf{x}-\mathbf{z}_{\tilde{y}}\right) \tilde{\mathbf{a}}(\tilde{\mathbf{y}}) d \sigma_{\tilde{y}} \\
& +\mathcal{O}\left(\delta^{2}\|\mathbf{x}\|^{-1}\|\tilde{\mathbf{a}}\|_{\mathrm{TH}^{-1 / 2}(\partial D)}\right) .
\end{aligned}
$$

With the above expansion, we can show the following theorem

Theorem 4.2. Let $\mathbf{E}_{\delta}$ be the solution to (1.14) with $D_{\delta}$ described in (4.2) and 4.3). Define $\tilde{\Phi}(\tilde{\mathbf{y}}):=\Phi(\mathbf{y})=\nu_{\mathbf{y}} \times\left.\mathbf{E}_{\delta}(\mathbf{y})\right|_{\partial D_{\delta}} ^{+}$, then there holds for $\mathbf{x} \in \mathbb{R}^{3} \backslash \bar{D}$

$$
\begin{aligned}
& \left(\mathbf{E}_{\delta}-\mathbf{E}^{i}\right)(\mathbf{x})=\int_{\Gamma_{0}} G_{\omega}\left(\mathbf{x}-\mathbf{z}_{\tilde{y}}\right) \mathbb{M}\left[\mathbf{n} \times \mathbf{E}^{i}(\mathbf{z})\right]\left(\mathbf{z}_{\tilde{y}}\right) d \sigma_{\mathbf{z}_{\tilde{y}}} \\
& +\mathcal{O}\left(\|\mathbf{x}\|^{-1}\left(\|\tilde{\Phi}\|_{\mathrm{TH}^{-1 / 2}\left(S^{0}\right)}+\delta\left(\|\tilde{\Phi}\|_{\mathrm{TH}^{-1 / 2}(\partial D)}+1\right)\right)\right),
\end{aligned}
$$

where

$$
\mathbb{M}:=\left(-\frac{I}{4}+\mathcal{M}_{\Gamma_{0}}^{\omega}\right)^{-1} \mathcal{M}_{\Gamma_{0}}^{\omega}\left(\frac{I}{4}+\mathcal{M}_{\Gamma_{0}}^{\omega}\right)^{-1}
$$

with $\mathcal{M}_{\Gamma_{0}}^{\omega}$ defined by

$$
\mathcal{M}_{\Gamma_{0}}^{\omega}[\Theta(\mathbf{z})]\left(\mathbf{z}_{\tilde{x}}\right):=\mathbf{n} \times \nabla_{\mathbf{z}_{\tilde{x}}} \times \int_{\Gamma_{0}} G_{\omega}\left(\mathbf{z}_{\tilde{x}}-\mathbf{z}_{\tilde{y}}\right) \Theta\left(\mathbf{z}_{\tilde{y}}\right) d \sigma_{\mathbf{z}_{\tilde{y}}} .
$$

Proof. We first recall that the solution to (1.14) can be represented by (2.7) and (2.8), where the density function a satisfies (2.9). Next, by using the fact that

$$
-\frac{I}{2}+\mathcal{M}_{S^{0}}^{\omega}: \mathrm{TH}^{-1 / 2}\left(S^{0}\right) \rightarrow \mathrm{TH}^{-1 / 2}\left(S^{0}\right)
$$


is invertible (cf. 33.36]), and using (2.9), (4.11), along with the use of the expansion of $\mathbf{E}^{i}$ in $\mathbf{z}$, one has by direct calculations that

$$
\begin{aligned}
\tilde{\mathbf{a}}(\tilde{\mathbf{y}})= & \left(-\frac{I}{2}+\mathcal{M}_{S^{0}}^{\omega}\right)^{-1}\left[\nu \times \mathbf{E}^{i}(\mathbf{z})+\tilde{\Phi}\right](\tilde{\mathbf{y}})+\mathcal{O}\left(\delta\left(\|\tilde{\Phi}\|_{\mathrm{TH}^{-1 / 2}(\partial D)}+1\right)\right) \\
= & \left(-\frac{I}{2}+\mathcal{M}_{S^{0}}^{\omega}\right)^{-1}\left[\nu \times \mathbf{E}^{i}(\mathbf{z})\right](\tilde{\mathbf{y}}) \\
& +\mathcal{O}\left(\|\tilde{\Phi}\|_{\mathrm{TH}^{-1 / 2}\left(S^{0}\right)}+\delta\left(\|\tilde{\Phi}\|_{\mathrm{TH}^{-1 / 2}(\partial D)}+1\right)\right)
\end{aligned}
$$

for all $\tilde{\mathbf{y}} \in S^{0} \cup S^{1}$. Noting that for $\tilde{\mathbf{y}} \in \Gamma^{1},(\tilde{\mathbf{y}}-2 \mathbf{n}) \in \Gamma^{2}$, we define $\tilde{\psi}(\tilde{\mathbf{y}}):=$ $\tilde{\mathbf{a}}(\tilde{\mathbf{y}}-2 \mathbf{n})$ for $\tilde{\mathbf{y}} \in \Gamma^{1}$ and $\tilde{\psi}(\tilde{\mathbf{y}}):=\tilde{\mathbf{a}}(\tilde{\mathbf{y}}+2 \mathbf{n})$ for $\tilde{\mathbf{y}} \in \Gamma^{2}$. By using the fact that

$$
\nu_{\tilde{\mathbf{y}}-2 n_{\tilde{\mathbf{y}}}}=-\nu_{\tilde{\mathbf{y}}}=-\mathbf{n} \quad \text { for } \quad \tilde{\mathbf{y}} \in \Gamma^{1},
$$

and the definition 4.10, one obtains (assuming for a while that $\tilde{\mathbf{x}} \in S^{0}$ )

$$
\begin{aligned}
\tilde{\mathbf{a}}(\tilde{\mathbf{y}}-2 \mathbf{n})=\tilde{\psi}(\tilde{\mathbf{y}})= & \left(\frac{I}{2}+\mathcal{M}_{S^{0}}^{\omega}\right)^{-1}\left[\mathbf{n} \times \mathbf{E}^{i}\left(\mathbf{z}_{\tilde{x}}\right)\right](\tilde{\mathbf{y}}) \\
& +\mathcal{O}\left(\|\mathbf{x}\|^{-1}\left(\|\tilde{\Phi}\|_{\mathrm{TH}^{-1 / 2}\left(S^{0}\right)}+\delta\left(\|\tilde{\Phi}\|_{\mathrm{TH}^{-1 / 2}(\partial D)}+1\right)\right)\right)
\end{aligned}
$$

for all $\tilde{\mathbf{y}} \in \Gamma^{1}$. By inserting (4.17) and (4.18) into (4.13), one then has that for $\mathbf{x} \in \mathbb{R}^{3} \backslash \bar{D}$

$$
\begin{aligned}
& \mathcal{S}_{D_{\delta}}^{\omega}[\mathbf{a}](\mathbf{x})=\int_{S^{0}} G_{\omega}\left(\mathbf{x}-\mathbf{z}_{\tilde{y}}\right) \tilde{\mathbf{a}}(\tilde{\mathbf{y}}) d \sigma_{\tilde{y}}+\mathcal{O}\left(\|\mathbf{x}\|^{-1}\left(\delta\left(\|\tilde{\Phi}\|_{\mathrm{TH}^{-1 / 2}(\partial D)}+1\right)\right)\right) \\
= & \int_{\Gamma^{1}} G_{\omega}\left(\mathbf{x}-\mathbf{z}_{\tilde{y}}\right)(\tilde{\mathbf{a}}(\tilde{\mathbf{y}})+\tilde{\psi}(\tilde{\mathbf{y}})) d \sigma_{\tilde{y}}++\mathcal{O}\left(\|\mathbf{x}\|^{-1}\left(\delta\left(\|\tilde{\Phi}\|_{\mathrm{TH}^{-1 / 2}(\partial D)}+1\right)\right)\right) \\
= & \int_{\Gamma^{1}} G_{\omega}\left(\mathbf{x}-\mathbf{z}_{\tilde{y}}\right) \hat{\mathbb{M}}\left[\mathbf{n} \times \mathbf{E}^{i}(\mathbf{z})\right](\tilde{\mathbf{y}}) d \sigma_{\tilde{y}} \\
& +\mathcal{O}\left(\|\mathbf{x}\|^{-1}\left(\|\tilde{\Phi}\|_{\mathrm{TH}^{-1 / 2}\left(S^{0}\right)}+\delta\left(\|\tilde{\Phi}\|_{\mathrm{TH}^{-1 / 2}(\partial D)}+1\right)\right)\right),
\end{aligned}
$$

where $\hat{\mathbb{M}}$ is defined by

$$
\hat{\mathbb{M}}:=2\left(-\frac{I}{2}+\mathcal{M}_{S^{0}}^{\omega}\right)^{-1} \mathcal{M}_{S^{0}}^{\omega}\left(\frac{I}{2}+\mathcal{M}_{S^{0}}^{\omega}\right)^{-1} .
$$

Since the function $\Theta(\mathbf{z}):=\mathbf{n} \times \mathbf{E}^{i}(\mathbf{z})$ depends only on $\mathbf{z} \in \Gamma_{0}$, and hence by definition 4.16 one readily verifies that

$$
\mathcal{M}_{\Gamma_{0}}^{\omega}[\Theta]\left(\mathbf{z}_{\tilde{x}}\right)=\frac{1}{2} \mathcal{M}_{S^{0}}^{\omega}[\hat{\Theta}(\tilde{\mathbf{y}})]\left(\mathbf{z}_{\tilde{x}}+\mathbf{n}\right), \quad \tilde{\mathbf{x}} \in \Gamma_{1},
$$

for $\hat{\Theta}(\tilde{\mathbf{y}})=\mathbf{n} \times \mathbf{E}^{i}\left(\mathbf{z}_{\tilde{y}}\right), \tilde{\mathbf{y}} \in S^{0}$. Therefore, we can replace the operator $\hat{\mathbb{M}}$ in 4.19 to be

$$
\mathbb{M}:=\left(-\frac{I}{4}+\mathcal{M}_{\Gamma_{0}}^{\omega}\right)^{-1} \mathcal{M}_{\Gamma_{0}}^{\omega}\left(\frac{I}{4}+\mathcal{M}_{\Gamma_{0}}^{\omega}\right)^{-1}
$$


and the proof is complete.

By using Theorem 4.2, we next consider a particular case by assuming that $\tilde{\Phi} \equiv 0$. Physically speaking, this corresponds to the case that $D_{\delta}$ is a so-called perfectly electric conductor. The result in the next theorem already partly reveals the partial-cloaking effect.

Theorem 4.3. Let $D_{\delta}$ be as described in (4.2) and (4.3). Consider the following scattering problem

$$
\begin{cases}\nabla \times \mathbf{E}_{\delta}^{p}-i \omega \mathbf{H}_{\delta}^{p}=0 & \text { in } \quad \mathbb{R}^{3} \backslash \bar{D}_{\delta} \\ \nabla \times \mathbf{H}_{\delta}^{p}+i \omega \mathbf{E}_{\delta}^{p}=0 & \text { in } \mathbb{R}^{3} \backslash \bar{D}_{\delta} \\ \nu \times\left.\mathbf{E}_{\delta}^{p}\right|_{+}=0 & \text { on } \quad \partial D_{\delta}\end{cases}
$$

subject to the Silver-Müller radiation condition:

$$
\lim _{|\mathbf{x}| \rightarrow \infty}\|\mathbf{x}\|\left(\left(\mathbf{H}_{\delta}^{p}-\mathbf{H}^{i}\right) \times \hat{\mathbf{x}}-\left(\mathbf{E}_{\delta}^{p}-\mathbf{E}^{i}\right)\right)=0 .
$$

Let $\mathbf{E}_{\infty}^{\delta}\left(\hat{\mathbf{x}} ; \mathbf{E}^{i}\right)$ be the corresponding scattering amplitude to 4.20 - (4.21). Then there holds

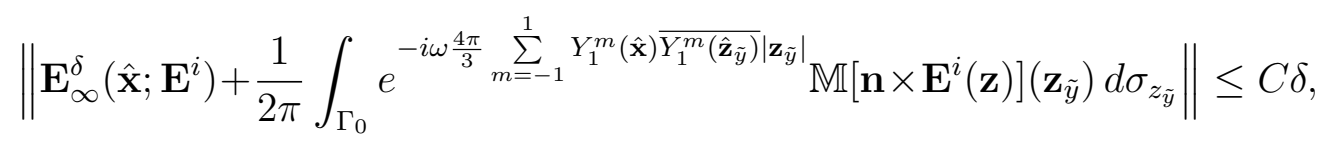

where $\mathbb{M}$ is defined in 4.15) and $C$ depends only on $\omega$ and D. Furthermore, if there holds

$$
\|\mathbf{n} \times \mathbf{p}\| \leq \epsilon, \quad \epsilon \ll 1,
$$

then for sufficient small $\delta \in \mathbb{R}_{+}$, one has

$$
\left\|\mathbf{E}_{\infty}^{\delta}\left(\hat{\mathbf{x}} ; \mathbf{E}^{i}\right)\right\| \leq C(\epsilon+\delta)
$$

where $C$ depends only on $\omega$ and $D$.

Proof. We start with the following addition formula (see, e.g., 33])

$$
\frac{1}{\|\mathbf{x}-\mathbf{y}\|}=4 \pi \sum_{n=0}^{\infty} \sum_{m=-n}^{n} \frac{1}{2 n+1} Y_{n}^{m}(\xi, \vartheta) \overline{Y_{n}^{m}\left(\xi^{\prime}, \vartheta^{\prime}\right)} \frac{q^{\prime n}}{q^{n+1}}
$$

where $(q, \xi, \vartheta)$ and $\left(q^{\prime}, \xi^{\prime}, \vartheta^{\prime}\right)$ are the spherical coordinates of $\mathbf{x}$ and $\mathbf{y}$, respectively; and $Y_{n}^{m}$ is the spherical harmonic function of degree $n$ and order $m$. For simplicity, the parameters $(q, \xi, \vartheta)$ and $\left(q^{\prime}, \xi^{\prime}, \vartheta^{\prime}\right)$ shall be replaced by $(\|\mathbf{x}\|, \hat{\mathbf{x}})$ and $(\|\mathbf{y}\|, \hat{\mathbf{y}})$, respectively. It then follows by 4.25 that

$$
\|\mathbf{x}-\mathbf{y}\|=\|\mathbf{x}\|\left(1-\frac{4 \pi}{3} \sum_{m=-1}^{1} Y_{1}^{m}(\hat{\mathbf{x}}) \overline{Y_{1}^{m}(\hat{\mathbf{y}})} \frac{\|\mathbf{y}\|}{\|\mathbf{x}\|}\right)+\mathcal{O}\left(\|\mathbf{x}\|^{-1}\right) .
$$

The solution $\mathbf{E}_{\delta}^{p}$ in 4.20 and 4.21 can be represented by

$$
\mathbf{E}_{\delta}^{p}=\mathbf{E}^{i}+\mathcal{A}_{D_{\delta}}^{\omega}[\mathbf{a}] \quad \text { in } \quad \mathbb{R}^{3} \backslash D_{\delta},
$$


with a satisfying

$$
\left(-\frac{I}{2}+\mathcal{M}_{D_{\delta}}^{\omega}\right)[\mathbf{a}](\mathbf{y})=-\nu_{\mathbf{y}} \times \mathbf{E}^{i}(\mathbf{y}), \quad \mathbf{y} \in \partial D_{\delta}
$$

Then one can easily derive from the expansion formula 4.14 with $\tilde{\Phi}=0$ that $\left(\mathbf{E}_{\delta}-\mathbf{E}^{i}\right)(\mathbf{x})=2 \int_{\Gamma_{0}} G_{\omega}\left(\mathbf{x}-\mathbf{z}_{\tilde{y}}\right) \mathbb{M}\left[\mathbf{n} \times \mathbf{E}^{i}(\mathbf{z})\right]\left(\mathbf{z}_{\tilde{y}}\right) d \sigma_{z_{\tilde{y}}}+\mathcal{O}\left(\|\mathbf{x}\|^{-1} \delta\right), \mathbf{x} \in \mathbb{R}^{3} \backslash D_{\delta}$,

where $\mathbb{M}$ is defined by 4.15$)$. By combining 4.27$)$ and $(4.26)$, we then have

$$
\mathbf{E}_{\infty}^{\delta}\left(\hat{\mathbf{x}} ; \mathbf{E}^{i}\right)=-\frac{1}{2 \pi} \int_{\Gamma_{0}} e^{-i \omega \frac{4 \pi}{3} \sum_{m=-1}^{1} Y_{1}^{m}(\hat{\mathbf{x}}) \overline{Y_{1}^{m}\left(\hat{\mathbf{z}}_{\tilde{y}}\right)}\left|\mathbf{z}_{\tilde{y}}\right|} \mathbb{M}\left[\mathbf{n} \times \mathbf{E}^{i}(\mathbf{z})\right]\left(\mathbf{z}_{\tilde{y}}\right) d \sigma_{z_{\tilde{y}}}+\mathcal{O}(\delta),
$$

which readily proves 4.22). Next by the definition of $\mathbb{M}$ in 4.15 , one can show that

$$
\left\|\mathbb{M}\left[\mathbf{n} \times \mathbf{E}^{i}(\mathbf{z})\right]\right\|_{\mathrm{TH}^{-1 / 2}\left(\Gamma_{0}\right)} \leq C\left\|\mathbf{n} \times \mathbf{E}^{i}(\mathbf{z})\right\|_{\mathrm{TH}^{-1 / 2}\left(\Gamma_{0}\right)},
$$

where $C$ depends only on $\Gamma_{0}$ and $\omega$. This together with (4.28) further implies that

$$
\left\|\mathbf{E}_{\infty}^{\delta}\left(\hat{\mathbf{x}} ; \mathbf{E}^{i}\right)\right\| \leq C\left(\left\|\mathbf{n} \times \mathbf{E}^{i}(\mathbf{z})\right\|_{\mathrm{TH}^{-1 / 2}\left(\Gamma_{0}\right)}+\delta\right) .
$$

Finally, by inserting the condition (4.23) into (4.29) and direct calculations, one can easily show 4.24).

The proof is complete.

4.2. Proof of Theorem 4.1. In this section, we present the proof of Theorem 4.1, which follows a similar spirit to those of Theorems 3.1 and 4.3 . The major idea is to control the norm $\|\tilde{\Phi}\|_{\mathrm{TH}^{-1 / 2}(\partial D)}$, which was taken to be identically zero in Theorem 4.3 .

Lemma 4.3. Let $\left(\mathbf{E}_{\delta}, \mathbf{H}_{\delta}\right)$ be the pair of solutions to (1.14) with $\left\{\Omega ; \varepsilon_{\delta}, \mu_{\delta}, \sigma_{\delta}\right\} \subset$ $\left\{\mathbb{R}^{3} ; \varepsilon_{\delta}, \mu_{\delta}, \sigma_{\delta}\right\}$ defined in (1.3) and $\left\{D_{\delta} \backslash \bar{D}_{\delta / 2} ; \varepsilon_{\delta}, \mu_{\delta}, \sigma_{\delta}\right\}$ given in (4.4). Then there holds the following estimates for $j=0,1,2$,

$\int_{D_{\delta}^{j} \backslash D_{\delta / 2}}\left\|\mathbf{E}_{\delta}\right\|^{2} d \mathbf{x} \leq C \delta^{j-t}\left(\left\|\nu \times \mathbf{E}_{\delta}^{+}\right\|_{\mathrm{TH}_{\mathrm{div}}^{-1 / 2}\left(\partial B_{R}\right)}^{2}+\|\tilde{\mathbf{a}}\|_{\mathrm{TH}^{-1 / 2}\left(S^{0}\right)}+\delta\|\tilde{\mathbf{a}}\|_{\mathrm{TH}^{-1 / 2}(\partial D)}\right)$, where the constant $C$ depends only on $R$ and $\omega$.

Proof. We first note that (3.41) still holds for the scattering problem described in the present lemma. Then one has

$$
\int_{D_{\delta} \backslash \bar{D}_{\delta / 2}} \sigma_{l} \mathbf{E}_{\delta} \cdot \overline{\mathbf{E}}_{\delta} d \mathbf{x} \leq C\left\|\nu \times \mathbf{E}_{\delta}^{+}\right\|_{\mathrm{TH}_{\mathrm{div}}^{-1 / 2}\left(\partial B_{R}\right)}\left\|\Lambda\left(\nu \times \mathbf{E}_{\delta}^{+}\right)\right\|_{\mathrm{TH}_{\mathrm{div}}^{-1 / 2}\left(\partial B_{R}\right)}+\mathbb{R}_{1}+\mathbb{R}_{2},
$$


where by using (2.8) and 4.12 one further has

$$
\begin{aligned}
\mathbb{R}_{1} & =\left.\left|\Re \int_{\partial D_{\delta}}\left(\nu \times \overline{\mathbf{E}^{i}}\right) \cdot\left(\nu \times \nu \times \mathbf{H}_{\delta}^{+}\right)\right|_{+} d \sigma_{x}|=| \Re \int_{\partial D_{\delta}} \overline{\mathbf{E}^{i}} \cdot\left(\nu \times \mathbf{H}_{\delta}^{+}\right)\right|_{+} d \sigma_{x} \mid \\
& \leq \frac{1}{\omega}\left|\int_{S^{0}} \overline{\mathbf{E}^{i}}\left(\mathbf{z}_{\tilde{x}}\right) \cdot \mathcal{L}_{S^{0}}^{\omega}[\tilde{\mathbf{a}}](\tilde{\mathbf{x}}) d \sigma_{\tilde{x}}\right|+\mathcal{O}\left(\delta\|\tilde{\mathbf{a}}\|_{\mathrm{TH}^{-1 / 2}(\partial D)}\right),
\end{aligned}
$$

and by using (4.11) one further has

$$
\begin{aligned}
\mathbb{R}_{2} & =\left.\left|\Re \int_{\partial D_{\delta}}\left(\nu \times \overline{\mathbf{E}_{\delta}^{+}}\right)\right|_{+} \cdot\left(\nu \times \nu \times \mathbf{H}^{i}\right) d \sigma_{x}|=| \Re \int_{\partial D_{\delta}} \overline{\mathbf{E}_{\delta}^{+}}\right|_{+} \cdot\left(\nu \times \mathbf{H}^{i}\right) d \sigma_{x} \mid \\
& \leq \delta\left|\int_{S^{0}} \mathcal{M}_{S^{0}}^{\omega}[\tilde{\mathbf{a}}](\tilde{\mathbf{x}}) \cdot \mathbf{H}^{i}\left(\mathbf{z}_{\tilde{x}}\right) d \sigma_{\tilde{x}}\right|+\mathcal{O}\left(\delta^{2}\|\tilde{\mathbf{a}}\|_{\mathrm{TH}^{-1 / 2}(\partial D)}\right) .
\end{aligned}
$$

Combining the above estimates with (4.5), (4.6) and the definition of $\sigma_{l}$ in (4.4), we can complete the proof.

Proof of Theorem 4.1. First, by using (4.14) and (4.7) one obtains

$$
\left\|\mathbf{A}_{\infty}^{\delta}(\hat{\mathbf{x}} ; \mathbf{p}, \mathbf{d})\right\| \leq C\left(\epsilon+\|\tilde{\Phi}\|_{\mathrm{TH}^{-1 / 2}\left(S^{0}\right)}+\delta\left(\|\tilde{\Phi}\|_{\mathrm{TH}^{-1 / 2}(\partial D)}+1\right)\right),
$$

where $\tilde{\Phi}(\tilde{\mathbf{x}})=\Phi(\mathbf{x}):=\nu \times\left.\mathbf{E}_{\delta}\right|_{+}(\mathbf{x})$ for $\mathbf{x} \in \partial D_{\delta}$. The following estimate can be obtained by using the result in Lemma 4.1 and a completely similar argument as that in the proof of Lemma 3.9.

$$
\|\tilde{\Phi}\|_{\mathrm{TH}^{-1 / 2}\left(S^{j}\right)} \leq C \delta^{-(j+1) / 2+\beta_{j}}\left\|\mathbf{E}_{\delta}\right\|_{L^{2}\left(D_{\delta}^{j} \backslash D_{\delta / 2}\right)^{3}}, \quad j=0,1,2,
$$

where $\beta_{j}=\min \{1,-j+r+s,-j+t+s\}, j=0,1,2$. On the other hand, by using 4.17), Lemma 4.3 and (4.30), one has

$$
\begin{aligned}
\int_{D_{\delta}^{j} \backslash D_{\delta / 2}}\left\|\mathbf{E}_{\delta}\right\|^{2} d \mathbf{x} \leq C \delta^{j-t} & \left(\|\tilde{\Phi}\|_{\mathrm{TH}^{-1 / 2}\left(S^{0}\right)}^{2}+\delta^{2}\|\tilde{\Phi}\|_{\mathrm{TH}^{-1 / 2}(\partial D)}^{2}\right. \\
+ & \left.\|\tilde{\Phi}\|_{\mathrm{TH}^{-1 / 2}\left(S^{0}\right)}+\delta\|\tilde{\Phi}\|_{\mathrm{TH}^{-1 / 2}(\partial D)}+\epsilon+\delta\right), \quad j=0,1,2 .
\end{aligned}
$$

Inserting 4.32) back into 4.31), one can show

$$
\begin{aligned}
\|\tilde{\Phi}\|_{\mathrm{TH}^{-1 / 2}\left(S^{j}\right)} \leq & C \delta^{\beta_{j}-t / 2-1 / 2}\left(\|\tilde{\Phi}\|_{\mathrm{TH}^{-1 / 2}\left(S^{0}\right)}+\delta^{1 / 2}\|\tilde{\Phi}\|_{\mathrm{TH}^{-1 / 2}(\partial D)}\right. \\
& \left.+\|\tilde{\Phi}\|_{\mathrm{TH}^{-1 / 2}\left(S^{0}\right)}^{1 / 2}+\epsilon^{1 / 2}+\delta^{1 / 2}\right), \quad j=0,1,2 .
\end{aligned}
$$

Noting that $\beta_{2} \leq \beta_{1} \leq \beta_{0}$ and $\beta_{2}-t / 2>0$, one has for $\delta \in \mathbb{R}_{+}$sufficiently small that

$$
\|\tilde{\Phi}\|_{\mathrm{TH}^{-1 / 2}(\partial D)} \leq C \delta^{\beta_{2}-t / 2-1 / 2}\left(\|\tilde{\Phi}\|_{\mathrm{TH}^{-1 / 2}\left(S^{0}\right)}+\|\tilde{\Phi}\|_{\mathrm{TH}^{-1 / 2}\left(S^{0}\right)}^{1 / 2}+\epsilon^{1 / 2}+\delta^{1 / 2}\right) .
$$

Inserting (4.34) back into (4.33) (for $j=0$ ), there holds

$$
\|\tilde{\Phi}\|_{\mathrm{TH}^{-1 / 2}\left(S^{0}\right)} \leq C \delta^{\beta_{0}-t / 2-1 / 2}\left(\|\tilde{\Phi}\|_{\mathrm{TH}^{-1 / 2}\left(S^{0}\right)}+\|\tilde{\Phi}\|_{\mathrm{TH}^{-1 / 2}\left(S^{0}\right)}^{1 / 2}+\epsilon^{1 / 2}+\delta^{1 / 2}\right) .
$$


By $\beta_{2}-t / 2>0$, it is directly verified that $\beta_{0}-t / 2 \geq 1$ and hence

$$
2\left(\beta_{0}-t / 2\right)-1 \geq \beta_{0}-t / 2 .
$$

Using (4.35) and (4.36), one readily infers for $\delta \in \mathbb{R}_{+}$sufficiently small that

$$
\|\tilde{\Phi}\|_{\mathrm{TH}^{-1 / 2}\left(S^{0}\right)} \leq C\left(\delta^{\beta_{0}-t / 2}+\epsilon\right) .
$$

Now by inserting (4.37) into 4.34), one has

$$
\|\tilde{\Phi}\|_{\mathrm{TH}^{-1 / 2}(\partial D)} \leq C \delta^{\beta_{2}-t / 2-1 / 2}\left(\delta^{1 / 2}+\epsilon^{1 / 2}\right) \leq C\left(\delta^{\beta_{2}-t / 2}+\delta^{2\left(\beta_{2}-t / 2\right)-1}+\epsilon\right) .
$$

Finally by plugging (4.37) and (4.38) into 4.30), we arrive at 4.8 .

The proof is complete.

\section{ACKNOWLEDGMENT}

The work of Y. Deng was partially supported by the Mathematics and Interdisciplinary Sciences Project, Central South University, China. The work of H. Liu was partially supported by Hong Kong RGC General Research Funds, HKBU 12302415 and 405513, and the NSF grant of China, No. 11371115. The work of G. Uhlmann was supported by NSF and the Academy of Finland.

\section{REFERENCES}

[1] R. A. Adams, Sobolev Spaces, Academic Press, New York, 1975.

[2] H. Ammari, Y. Deng, P. Millien, Surface plasmon resonance of nanoparticles and applications in imaging, Archive for Rational Mechanics and Analysis, in press, 2015.

[3] H. Ammari, H. Kang, H. Lee and M. Lim, Enhancement of approximate-cloaking using generalized polarization tensors vanishing structures. Part I: The conductivity problem, Comm. Math. Phys., 317 (2013), 253-266.

[4] H. Ammari, H. Kang, H. Lee and M. Lim, Enhancement of approximate-cloaking. Part II: The Helmholtz equation, Comm. Math. Phys., 317 (2013), 485-502.

[5] H. Ammari, H. Kang, H. Lee and M. Lim, Enhancement of approximate cloaking for the full Maxwell equations, SIAM J. Appl. Math.,73 (2013), 2055-2076.

[6] G. Bao and H. Liu, Nearly cloaking the electromagnetic fields, SIAM J. Appl. Math., 74 (2014), 724-742.

[7] G. Bao, H. Liu and J. Zou, Nearly cloaking the full Maxwell equations: cloaking active contents with general conducting layers, J. Math. Pures Appl. (9), 101 (2014), 716-733.

[8] H. Chen and C. T. Chan, Acoustic cloaking and transformation acoustics, J. Phys. D: Appl. Phys., 43 (2010), 113001.

[9] Y. Deng, H. Liu and G. Uhlmann, On regularized full- and partial-cloaks in acoustic scattering, preprint, arXiv:1502.01174

[10] D. Colton and R. Kress, Inverse Acoustic and Electromagnetic Scattering Theory, 2nd Edition, Springer-Verlag, Berlin, 1998.

[11] A. Greenleaf, Y. Kurylev, M. Lassas and G. Uhlmann, Improvement of cylindrical cloaking with SHS lining, Optics Express, 15 (2007), 12717-12734.

[12] A. Greenleaf, Y. Kurylev, M. Lassas and G. Uhlmann, Full-wave invisibility of active devices at all frequencies, Comm. Math. Phys., 279 (2007), 749-789.

[13] A. Greenleaf, Y. Kurylev, M. Lassas and G. Uhlmann, Isotropic transformation optics: approximate acoustic and quantum cloaking, New J. Phys., 10 (2008), 115024. 
[14] A. Greenleaf, Y. Kurylev, M. Lassas and G. Uhlmann, Invisibility and inverse problems, Bulletin A. M. S., 46 (2009), 55-97.

[15] A. Greenleaf, Y. Kurylev, M. Lassas and G. Uhlmann, Cloaking devices, electromagnetic wormholes and transformation optics, SIAM Review, 51 (2009), 3-33.

[16] A. Greenleaf, M. Lassas and G. Uhlmann, Anisotropic conductivities that cannot be detected by EIT, Physiolog. Meas, (special issue on Impedance Tomography), 24 (2003), 413.

[17] A. Greenleaf, M. Lassas and G. Uhlmann, On nonuniqueness for Calderón's inverse problem, Math. Res. Lett., 10 (2003), 685-693.

[18] R. Griesmaier, An asymptotic factorization method for inverse electromagnetic scattering in layered media, SIAM J. Appl. Math., 68 (2008), 1378C1403.

[19] C. Hazard and M. Lenoir, On the solution of time-harmonic scattering problems for Maxwell's equations, SIAM J. Math. Anal., 27 (1996), 1597-1630.

[20] R. Kohn, O. Onofrei, M. Vogelius and M. Weinstein, Cloaking via change of variables for the Helmholtz equation, Comm. Pure Appl. Math., 63 (2010), 973-1016.

[21] R. Kohn, H. Shen, M. Vogelius and M. Weinstein, Cloaking via change of variables in electrical impedance tomography, Inverse Problems, 24 (2008), 015016.

[22] R. Leis, Zur Theorie elektromagnetischer Schwingungen in anisotropen inhomogenene Medien, Math. Z., 106 (1968), 213-224.

[23] R. Leis, Initial Boundary Value Problems in Mathematical Physics, Teubner, Stuttgart; Wiley, Chichester, 1986.

[24] U. Leonhardt, Optical conformal mapping, Science, 312 (2006), 1777-1780.

[25] J. Li, H. Liu, L. Rondi and G. Uhlmann, Regularized transformation-optics cloaking for the Helmholtz equation: from partial cloak to full cloak, Comm. Math. Phys., 335 (2015), $671-712$.

[26] J.L. Lions and E. Magenes, Non-Homogeneous Boundary Value Problems and Applications I, Springer-Verlag, 1970.

[27] H. Liu, Virtual reshaping and invisibility in obstacle scattering, Inverse Problems, 25 (2009), 045006.

[28] H. Liu and H. Sun, Enhanced approximate-cloak by FSH lining, J. Math. Pures Appl. (9), 99 (2013), 17-42.

[29] H. Liu and G. Uhmann, Regularized transformation-optics cloaking in acoustic and electromagnetic scattering, Book chapter edited by Societe Mathematique de France, in press, 2015.

[30] H. Liu and T. Zhou, Two dimensional invisibility cloaking by transformation optics, Discrete Contin. Dyn. Syst., 31 (2011), 525-543.

[31] J. C. Nédélec, Acoustic and Electromagnetic Equations: Integral Representations for Harmonic Problems, Springer-Verlag, New York, 2001.

[32] P. Monk, Finite ElementMethods for Maxwells Equations, Clarendon Press, Oxford, 2003.

[33] J.-C. Nédélec, Acoustic and Electromagnetic Equations. Integral Representations for Harmonic Problems, Applied Mathematical Sciences, Vol. 144, Springer-Verlag, New-York, 2001.

[34] J. B. Pendry, D. Schurig and D. R. Smith, Controlling electromagnetic fields, Science, 312 (2006), 1780-1782.

[35] J. Wloka, Partial Differential Equations, Cambridge University Press, Cambridge, 1987.

[36] M. E. Taylor, Partial Differential Equations II: Qualitative Studies of Liapproximate Equations, 2nd Edition, Springer, New York, 2011.

[37] G. Uhlmann, Visibility and invisibility, ICIAM 07-6th International Congress on Industrial and Applied Mathematics, Eur. Math. Soc., Zürich, pp. 381-408, 2009. 
School of Mathematics and Statistics, Central South University, Changsha, Hunan, P. R. China.

E-mail address: youjundeng@csu.edu.cn, dengyijun_001@163.com

Department of Mathematics, Hong Kong Baptist University, Kowloon, Hong KONG SAR.

E-mail address: hongyu.liuip@gmail.com

Department of Mathematics, University of Washington, Seattle, WA 98195, USA.

AND

Institute for Advanced Study, Hong Kong University of Science and Technology, Hong Kong SAR.

E-mail address: gunther@math.washington.edu 